\title{
WestVirginiaUniversity
}

THE RESEARCH REPOSITORY @ WVU

Graduate Theses, Dissertations, and Problem Reports

2014

\section{Numerical Modeling of Inertial Flows in Proppant-Reservoir Rock Interfaces}

Abdulla Alkaabi

Follow this and additional works at: https://researchrepository.wvu.edu/etd

\section{Recommended Citation}

Alkaabi, Abdulla, "Numerical Modeling of Inertial Flows in Proppant-Reservoir Rock Interfaces" (2014). Graduate Theses, Dissertations, and Problem Reports. 5069.

https://researchrepository.wvu.edu/etd/5069

This Thesis is protected by copyright and/or related rights. It has been brought to you by the The Research Repository @ WVU with permission from the rights-holder(s). You are free to use this Thesis in any way that is permitted by the copyright and related rights legislation that applies to your use. For other uses you must obtain permission from the rights-holder(s) directly, unless additional rights are indicated by a Creative Commons license in the record and/ or on the work itself. This Thesis has been accepted for inclusion in WVU Graduate Theses, Dissertations, and Problem Reports collection by an authorized administrator of The Research Repository @ WVU. For more information, please contact researchrepository@mail.wvu.edu. 


\title{
Numerical Modeling of Inertial Flows in Proppant-Reservoir Rock Interfaces
}

\author{
by \\ Abdulla Alkaabi \\ Thesis submitted to the \\ College of Engineering and Mineral Resources \\ at West Virginia University \\ in partial fulfillment of the requirements \\ for the degree of \\ Master of Science \\ in \\ Petroleum and Natural Gas Engineering \\ Ebrahim Fathi, Ph.D. \\ H. Ilkin Bilgesu, Ph.D \\ Ali Takbiri Borujeni, Ph.D., Chair \\ Petroleum and Natural Gas Engineering \\ Morgantown, West Virginia \\ 2014
}

Keywords: Lattice Boltzmann Modeling, Simulation, Non-Darcy, Non-Darcy Factor, Inertial Flow

Copyright 2014 Abdulla Alkaabi 


\author{
Abstract \\ Numerical Modeling of Inertial Flows in Proppant-Reservoir Rock Interfaces \\ by \\ Abdulla Alkaabi \\ Master of Science in Petroleum and Natural Gas Engineering \\ West Virginia University \\ Ali Takbiri Borujeni, Ph.D., Chair
}

Predicting accurate pressure drops in the reservoirs is essential for estimating the ultimate hydrocarbons recoveries and production rates. In hydraulically fractured wells, inertial flows can cause excessive pressure drops, beyond the predicted values form the Darcy equation. Therefore, predicting these excessive pressure drops through defining non-Darcy factors is of particular significance.

Excessive pressure drops in inertial flows are caused by acceleration/deceleration of fluids, which usually occur when fluids are moving from constricted areas to larger pores and vice versa. In the interface between the propped fracture and the reservoir rock, the pores in the latter are in connection with the former that can generate eddies and thus fluid acceleration/deceleration.

In this work, two-dimensional geometries are generated by combining coarse and tight porous media and their hydraulic properties, i.e., absolute permeability and non-Darcy factors, are calculated using lattice Boltzmann simulations. Based on the simulation results, calculated absolute permeability of generated porous media follows the harmonic averaging theory for flow through series of constituting porous media. However, the non-Darcy factor for the generated geometries are higher than the constituting geometries, which does not conform to any averaging approach. This affirms the common knowledge that non-Darcy factor is a property that cannot be upscaled. The results in this study broadens our knowledge of fluid flow in hydraulic fractures. 


\section{Acknowledgements}

I want to express my most sincere gratitude to my adviser Dr. Ali Takbiri Borujeni for giving me the opportunity to work under his advising and for spending the time in providing me with the necessary knowledge and the necessary resources in order to successfully complete this work. Moreover, I would like to acknowledge my committee members Ebrahim Fathi, Ph.D and H.Ilkin Bilgesu, Ph.D for their contribution and for critiquing this work which gave the final shape of this project. Furthermore, I want to express my gratitude to faculty and staff of the Department of Petroleum and Natural Gas Engineering at the College of Engineering and Mineral Resources at West Virginia University, for giving me the opportunity to conduct my graduate studies from which I gained more valuable knowledge while carrying out this work.

I want to mention the computer language Python (www.python.org) due to the fact that it was used to complete this work in writing LBM algorithm. In addition to that, I would like to express my appreciativeness to my sponsor company ADNOC (Abu Dhabi National Oil Company) for giving me the opportunity to complete my graduate studies and for providing me with the financial support to complete this project and my graduate studies.

Last but not least, I would love to express my gratefulness to my family and siblings who provided me with the courage and the moral support to complete this work and my graduate studies because they have been always backing me up with the support and inspiration in order to successfully finish my graduate studies. 


\section{Contents}

$\begin{array}{ll}\text { Acknowledgements } & \text { iii }\end{array}$

$\begin{array}{ll}\text { List of Figures } & \text { v }\end{array}$

List of Tables $\quad$ vii

Notation $\quad$ viii

1 Introduction $\quad 1$

1.1 Problem Statement . . . . . . . . . . . . . . . . . . . . 1

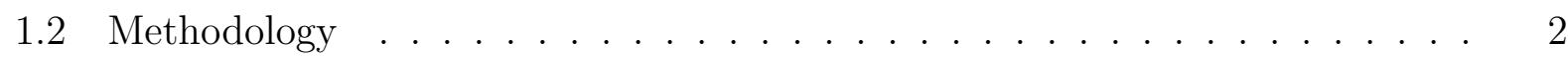

1.3 Background and Fundamentals of Fluid Flow in Porous Media . . . . . . . . 2

1.4 Criteria for Onset of Inertial Flow . . . . . . . . . . . . . . . . 3

2 Theory of Lattice Boltzmann $\quad 5$

2.1 Lattice Boltzmann Method $(\mathrm{LBM}) \ldots \ldots \ldots \ldots$

2.2 Boundary Conditions . . . . . . . . . . . . . . . 7

$\begin{array}{llr}3 & \text { Verification } & 8\end{array}$

4 Results $\quad 10$

4.1 Overview . . . . . . . . . . . . . . . . . . . . . 10

4.2 Numerical Simulation Results . . . . . . . . . . . . . . . . . . . . 11

4.3 Discussion . . . . . . . . . . . . . . . . . . . . 28

5 Conclusions and Recommendations $\quad 30$

5.1 Conclusions and Recommendations . . . . . . . . . . . . . . . . . . 30

5.2 Recommendations . . . . . . . . . . . . . . . . . . . . 31

$\begin{array}{ll}\text { References } & 32\end{array}$ 


\section{List of Figures}

2.1 Lattice Boltzmann Model D2Q9 . . . . . . . . . . . . . . . . . 5

3.1 Analytical Solution . . . . . . . . . . . . . . . . . . 9 9

4.1 Representation of combination of two circle packs with different circle sizes connected with each other to make a combined geometry $(1 \times 1)$ and $(2 \times 2)$ circle pack . . . . . . . . . . . . . . . . . . . 11

4.2 Simulations results for a single circle . . . . . . . . . . . . . . . . 12

4.3 Velocity streamlines for a single circle $F_{o}=9.97 \times 10^{-5}$ (left) and $F_{o}=$ $1.80 \times 10^{-1}$ (right) . . . . . . . . . . . . . . . . . . . 13

4.4 Simulations results for $(2 \times 2)$ circle pack . . . . . . . . . . . . 13

4.5 Velocity streamlines for $(2 \times 2)$ circle pack $F_{o}=3.09 \times 10^{-3}$ (left) and $F_{o}=$ $2.12 \times 10^{-1}$ (right) . . . . . . . . . . . . . . . . 14

4.6 Simulations results for $(3 \times 3)$ circle pack . . . . . . . . . . . . . 15

4.7 Velocity streamlines for $(3 \times 3)$ circle pack $F_{o}=1.24 \times 10^{-5}$ (left) and $F_{o}=$

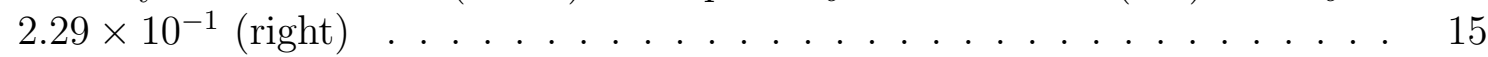

4.8 Simulations results for $(4 \times 4)$ circle pack . . . . . . . . . . . . 16

4.9 Velocity streamlines for $(4 \times 4)$ circle pack $F_{o}=8.23 \times 10^{-4}$ (left) and $F_{o}=$ $2.70 \times 10^{-1}$ (right) . . . . . . . . . . . . . . . 16

4.10 Simulations results for $(1 \times 1)+(2 \times 2)$ circle pack . . . . . . . . . 17

4.11 Velocity streamlines for $(1 \times 1)+(2 \times 2)$ circle pack $F_{o}=1.24 \times 10^{-3}$ (left) and $F_{o}=4.43 \times 10^{-1}$ (right) $\ldots \ldots \ldots \ldots 18$

4.12 Simulations results for $(1 \times 1)+(3 \times 3)$ circle pack . . . . . . . . . . 18

4.13 Velocity streamlines for $(1 \times 1)+(3 \times 3)$ circle pack $F_{o}=4.83 \times 10^{-5}$ (left)

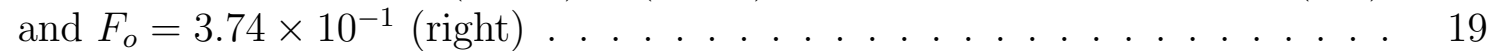

4.14 Simulations results for $(1 \times 1)+(4 \times 4)$ circle pack . . . . . . . . . 19

4.15 Velocity streamlines for $(1 \times 1)+(4 \times 4)$ circle pack $F_{o}=1.85 \times 10^{-3}(\mathrm{left})$ and $F_{o}=2.53 \times 10^{-1}$ (right) $\ldots \ldots \ldots \ldots \ldots$

4.16 Simulations results for $(2 \times 2)+(3 \times 3)$ circle pack . . . . . . . . . . 20

4.17 Velocity streamlines for $(2 \times 2)+(3 \times 3)$ circle pack $F_{o}=2.62 \times 10^{-5}$ (left)

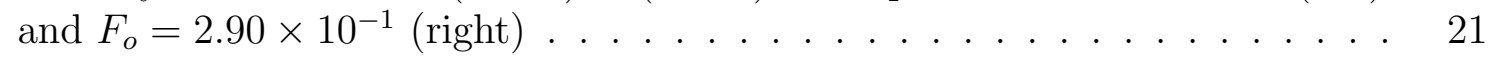

4.18 Simulations results for $(2 \times 2)+(4 \times 4)$ circle pack . . . . . . . . . . . 22

4.19 Velocity streamlines for $(2 \times 2)+(4 \times 4)$ circle pack $F_{o}=1.29 \times 10^{-5}$ (left)

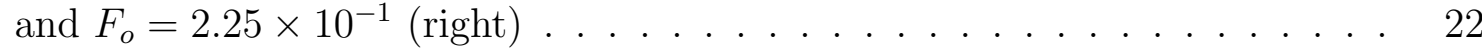


4.20 Simulations results for $(3 \times 3)+(4 \times 4)$ circle pack . . . . . . . . . . . 23

4.21 Velocity streamlines for $(3 \times 3)+(4 \times 4)$ circle pack $F_{o}=9.75 \times 10^{-6}(\mathrm{left})$ and $F_{o}=2.27 \times 10^{-1}$ (right) . . . . . . . . . . . . . . 23

4.22 Simulations results of 2-dimensional random porous media for 0.64 porosity . 24

4.23 Velocity streamlines for porosity $(0.64)$ random $2 \mathrm{D}$ porous media $F_{o}=4.87 \times$ $10^{-3}$ (left) and $F_{o}=1.96 \times 10^{-1}$ (right) . . . . . . . . . . . 25

4.24 Simulations results of 2-dimensional random porous media for 0.87 porosity .25

4.25 Velocity streamlines for porosity (0.87) random $2 \mathrm{D}$ porous media $F_{o}=1.42 \times$ $10^{-4}$ (left) and $F_{o}=7.89 \times 10^{-1}$ (right) . . . . . . . . . . . 26

4.26 Simulations results of 2-dimensional random porous media for low and high porosity . . . . . . . . . . . . . . . . . . 27

4.27 Velocity streamlines for low and high porosity $2 \mathrm{D}$ random porous media $F_{o}=$ $1.03 \times 10^{-4}$ (left) and $F_{o}=2.01 \times 10^{-1}$ (right) . . . . . . . . . . . 27

4.28 Eddies generated at the interface between fine and coarse geometries . . . . 29 


\section{List of Tables}

4.1 Summary of the LBM simulation results . . . . . . . . . . . . . . . 28 


\section{Notation}

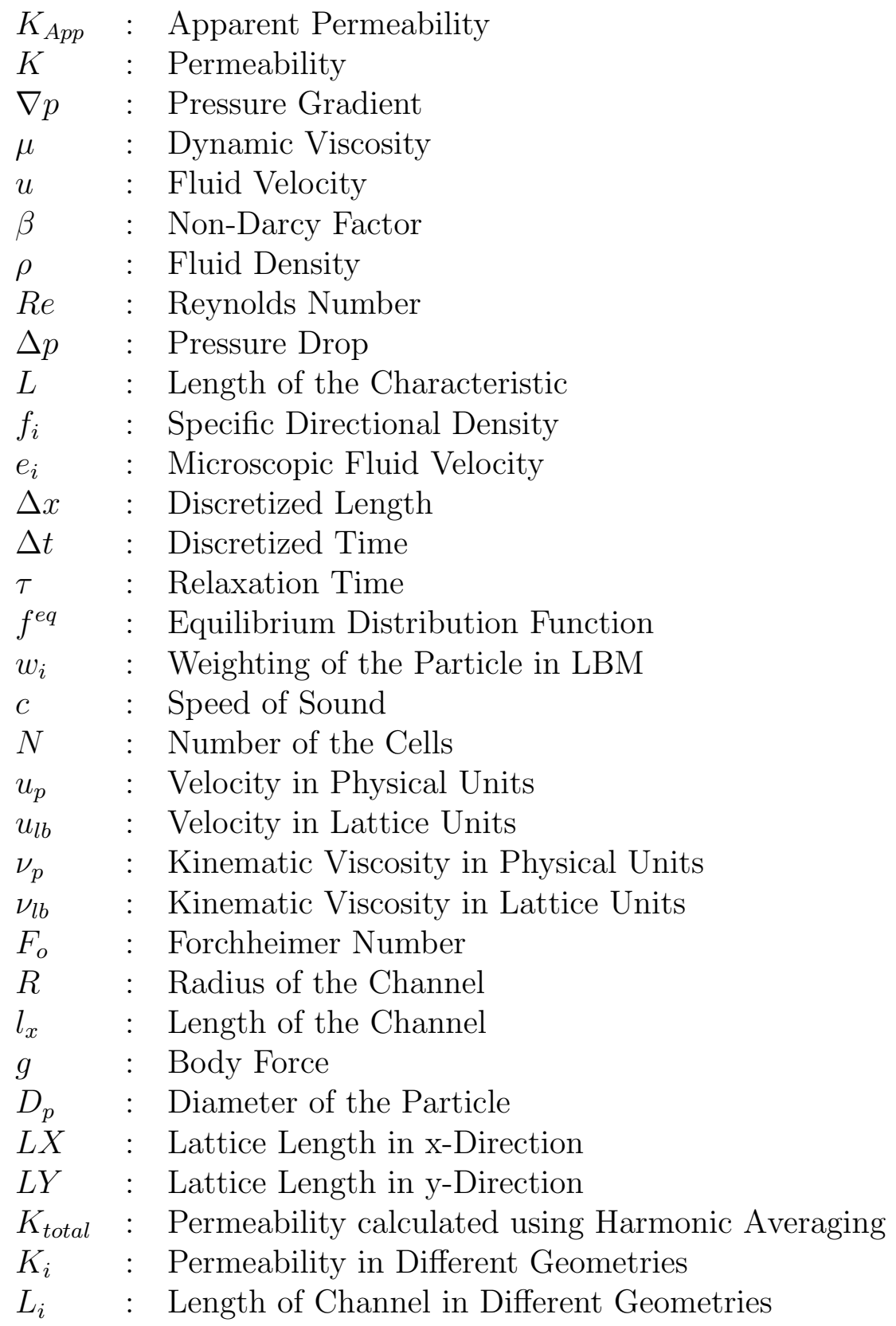




\section{Chapter 1}

\section{Introduction}

\subsection{Problem Statement}

The Darcy equation describes the flow of single phase fluids in porous media at low Reynolds numbers $(\operatorname{Re}<1)$ and the permeability is the constant of the proportionality in the linear relationship between the pressure gradient and the flow rate [1]. Deviations from this linear relationship at high Reynolds numbers $(R e>1)$ where inertial force becomes significant, are called non-Darcy or inertial flows [2]. The deviation from Darcy's flow commonly occurs at regions with high fluid velocities, such as at the region near the well-bore and hydraulic fractures. In these regions, the acceleration/deceleration of the fluids causes in the inertial force to increase and that can cause excessive pressure drops. Deviations from Darcy's equation can cause errors in reservoir modeling when attempting to history match the production data. Previous studies proved that the non-Darcy flows reduce the well and reservoir productivity index up to 20 times their original values (Holditch and Morse 1976)[3]. A separate study by Guppy at 1982 showed that the non-Darcy flow can significantly reduce the productivity index of a hydraulically fractured gas wells up to 50 percent from the original values [4].

The main objective of this work is to check if the properties of the non-Darcy flow can be upscaled. That will provide more understanding about the behavior of the flow around the well bore especially at hydraulically fractured wells. 


\subsection{Methodology}

There are some methods to calculate the permeability and non-Darcy parameters, such as flow experiments, analytical methods, empirical relationships, and numerical techniques. The experimental approach is a reliable methodology; however, it is expensive and time consuming. The analytical approach is based on applying ideal conditions into analytical expressions which may not be practical for real cases. The empirical approaches are difficult to apply to wide variety of porous media. However, the current methods are based on obtaning the flow properties of the proppant pack separately and to obtain the flow properties of the reservoir rock separately.

For this work, the methodology is to obtain the permeability and non-Darcy factor of the complex geometries created by combining simple 2 dimensional geometries. The flow simulations are conducted using Lattice Boltzmann Method (LBM). Lattice Boltzmann method (LBM) has been proved to be a reliable tool for numerical fluid flow problem [5]. LBM is widely used by researchers for complex fluid flow problems for a single phase or multiphase for different geometries, and variety of different boundary conditions for different fluid flow problems $[6,7]$.

\subsection{Background and Fundamentals of Fluid Flow in Porous Media}

Henry Darcy in 1856 [8], conducted an experiment for flow of water through vertical sand beds. He found that for flow of the fluid in laminar region, there is a linear relationship between flow rate and the pressure gradient. Based on this observation, he proposed the following equation for flow of single phase fluid in porous media:

$$
-\nabla p=\frac{\mu u}{K}
$$

where $\nabla p$ is the pressure gradient, $\mu$ is the viscosity, $u$ is fluid flow velocity and $K$ is the permeability, a property of porous media to transmit fluids through. 
At high fluid velocities, pressure gradient is not proportional to the flow rate and there are excessive pressure drops in porous media. Geertsma at 1974 stated that the reason for the excessive pressure drops is the present of the inertial flows [9]. Inertial flows are caused by acceleration/deceleration of fluids at regions with high fluid velocities $(R e>1)$.

One of the equations that was proposed to predict the fluid flow at high fluid rates is Forchheimer equation. According to Forcheimer, at high flow rates the pressure gradient is proportional to the square of the fluid velocity:

$$
-\nabla p=\frac{u \mu}{K}+\beta \rho u^{2}
$$

where $\rho$ is fluid density, and $\beta$ is the non-Darcy factor. In order to calculate the non-Darcy factor in numerical simulations, the apparent permeability is defined by rearranging the Forchheimer equation.

$$
\frac{1}{K_{a p p}}=\frac{1}{K}+\beta \frac{\rho|u|}{\mu}
$$

where the $|u|$ is the fluid velocity. By plotting the inverse of $K_{a p p}$ against fluid velocity $u$, non-Darcy factor can be calculated as the slope of the line.

\subsection{Criteria for Onset of Inertial Flow}

One of non-Darcy criteria is based on the Reynolds number where the values are between 1 - 100, the following equation represents Reynolds number:

$$
R e=\frac{\rho D_{p} u}{\mu}
$$

where $\rho$ is the density of the fluid, $D_{p}$ is the diameter of the particles, $u$ is the velocity of the fluid, and $\mu$ is the viscosity of the fluid[10].

Another non-Darcy criteria is based on the Forchheimner number where the values are between $0.005-0.2$, the following equation is the formula for Forchheimer number.

$$
F_{o}=\frac{K \beta u \rho}{\mu}
$$


where $F_{o}$ is the Forchheimer number, $K$ is the permeability, $\beta$ is the Non-Darcy factor, $u$ is the velocity of the fluid flow, $\rho$ is the density of the fluid and $\mu$ is the fluid viscosity.

The Forchhiemer number is usually used in numerical models [10] because it is advantageous over the Reynolds number due to the fact that its parameters are relatively simpler to be obtained than those in the Reynolds number, especially the particles diameter in the Reynolds number, $D_{p}$, which is one of the challenging parameters to be obtained. In Forchheimer number particle diameter was substituted in by product of permeability and non-Darcy factor, $\beta K$. 


\section{Chapter 2}

\section{Theory of Lattice Boltzmann}

\subsection{Lattice Boltzmann Method (LBM)}

LBM is a simplification of Boltzmann Equation in which we limit the position and directions at which fluids can move from continuum into handful, time is also discretized into time steps [5].

There are models such as D3Q19 and D2Q9 where $D$ is the dimension and $Q$ is the number of the directions a particle can travel. Figure 2.1 shows the schematic representation of $D 2 Q 9$ where $f_{(0-8)}$ are the directional densities.

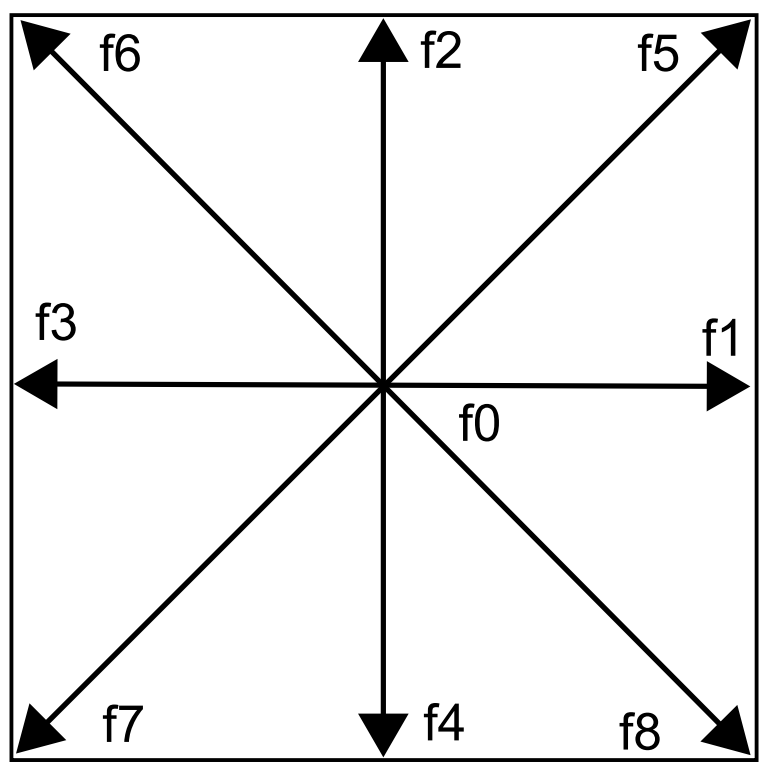

Figure 2.1: Lattice Boltzmann Model D2Q9 
There are two main steps in LBM: streaming and collision. In streaming, the specific directional densities $f_{i}$ travel to their near neighboring nodes. The following equation is a mathematical representation for the streaming step,

$$
f_{i}\left(x+e_{i} \Delta t, t+\Delta t\right)=f_{i}(x, t)
$$

where $e_{i}$ is the direction which the specific directional dentistry can move to its neighboring node, $f_{i}$ is the distribution function and $\Delta t$ is the discrete time step.

In the collision step, once the specific directional densities $f_{i}$ collide with their neighboring nodes, they tend to reach an equilibrium by exchanging the momentum between the particles. The following equation represent combined streaming of specific directional densities with a single relaxation time collision (BGK):

$$
f_{i}\left(x+e_{i} \Delta t, t+\Delta t\right)=f_{i}(x, t)-\frac{\left[f_{i}(x, t)-f_{i}^{e q}(x, t)\right]}{\tau},
$$

where $-\frac{\left[f_{i}(x, t)-f_{i}^{e q}(x, t)\right]}{\tau}$ is the collision part, $\tau$ is the relaxation time toward equilibrium and $f^{e q}$ is the equilibrium distribution function which represented as in the following equation:

$$
f_{i}^{e q}=w_{i} \rho(x)\left[1+3 \frac{e_{i} \cdot u}{c^{2}}+\frac{9}{2} \frac{\left(e_{i} \cdot u\right)^{2}}{c^{4}}-\frac{3}{2} \frac{u^{2}}{c^{2}}\right]
$$

where $w_{i}$ is the weighting for the particles; for instance D2Q9, the value $\frac{4}{9}$ is the weight for the particle at rest, $\frac{1}{9}$ is the particle weight at directions $(1,2,3$, and 4$)$ while $\frac{1}{36}$ is the particle weight for the directions $(5,6,7$, and 8$)$. The variable $c$ is the speed of the sound at lattice unit $\left(\frac{l u}{t s}\right)$, which is $\frac{1}{\sqrt{3}}$ and $u$ is the macroscopic velocity of the particles.

Macroscopic variables can be calculated using momenta of the directional densities, for instance, fluid density can be calculated using first moment and fluid velocity using the second moment of the directional densities:

$$
\begin{gathered}
\rho=\sum_{i=0}^{8} f_{i}, \\
u=\frac{1}{\rho} \sum_{i=0}^{8} f_{i} e_{i},
\end{gathered}
$$

where the $u$ is the macroscopic fluid velocity and $\rho$ is the macroscopic fluid density. 


\subsection{Boundary Conditions}

There are two different types of boundary condictions used in this work: bounceback and periodic. Bounceback is used at the solid-void interface to mimic no-slip boundary condition. Bounceback is widely used in LBM due to its simplicity [11]. When a particle reaches a solid node, the magnitude of the velocity of the particle stays the same; however, the direction of the velocity is reflected back to the fluid.

Periodic boundary condition is usually used as the inlet and outlet boundaries. When the distribution function reaches the outlet, the same distribution function comes back in the inlet and vice versa [5]. 


\section{Chapter 3}

\section{Verification}

It is necessary to verify the LBM simulation results with analytical solution of the problem. One of the simplest ways to verify the results is to compare them against Poiseuille equation for two dimensional channel.

$$
u_{x}(y)=\frac{1}{2 \nu} \frac{\Delta P}{l_{x}}\left(R^{2}-y^{2}\right)
$$

where the $u_{x}$ is the flow velocity values along the $x$ axis and a function of the $y$-coordinate, $\nu$ is the kinematic viscosity of fluid, $\Delta P$ is the pressure drop, $R$ is the radius of the channel and $l_{x}$ is the length of the channel. It is necessary to mention that $\frac{\Delta P}{l_{x}}$ is the same as the body force in LBM simulation. The parameter are converted from the lattice units to the physical units.

The following problem set is used for the verification:

$$
\begin{array}{ll}
L X \quad: & \text { lattice length } \mathrm{x} \text {-direction }=20 l u \\
L Y & : \text { lattice length y-direction }=20 l u \\
g & : \text { body force }=0.001 \frac{l u}{l t^{2}} \\
\nu \quad: \quad \text { kinematic viscosity }=\frac{1}{6} \frac{l t}{l u^{2}}
\end{array}
$$

these parameters are converted from lattice units to physical units.

$$
\begin{array}{ll}
R & : \text { the radius of the channel }=0.005 \mathrm{~m} \\
g & : \text { body force }=0.209868044 \frac{\mathrm{m}}{\mathrm{s}^{2}} \\
\nu & : \text { kinematic viscosity of water }=10^{-6} \mathrm{Pa.s}
\end{array}
$$

Figure 3.1 shows the velocity profiles of the Poiseuille solution versus the numerical 
solution which is obtained from the simulation of LBM. The figure shows that the LBM results are in agreement with the analytical Poiseuille flow for 2 dimensional channel.

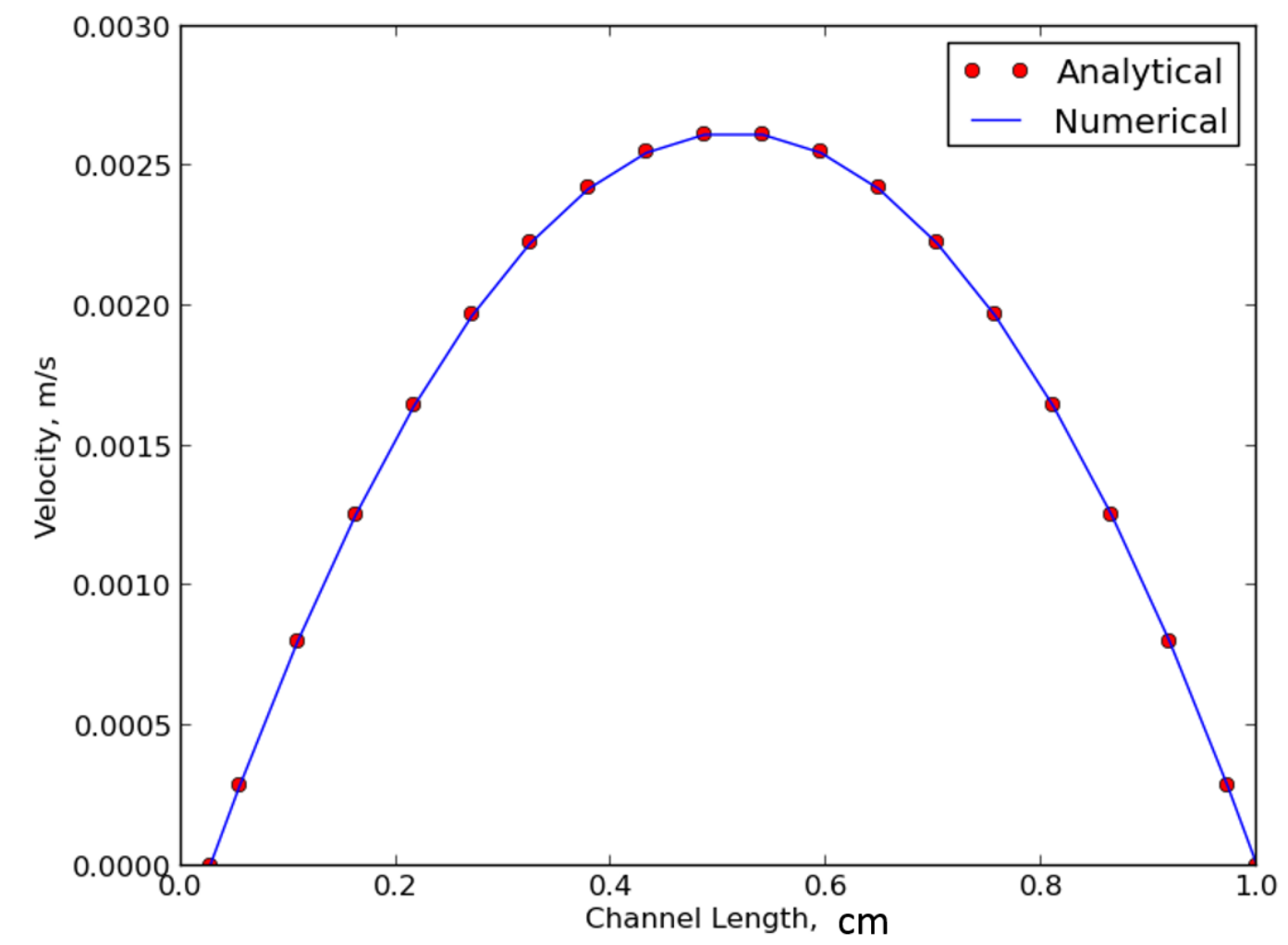

Figure 3.1: Analytical Solution

Figure 3.1 Verification of the LBM simulation results against Poiseuille equation for a two dimensional channel. 


\section{Chapter 4}

\section{Results}

\subsection{Overview}

In hydraulic fractured wells, proppant are used to keep the fracture open and to create a high conductivity flow path for the fluid, in the reservoir to flow towards the well-bore. The high velocity of the fluids, in the fracture can cause excessive pressure drops, where the inertial force becomes important and causes the relationship between the pressure drops and the fluid velocity to become non-linear [12]. These excessive pressure drops are due to the fluid decelerating from smaller pores through larger ones and accelerating from larger pores though smaller ones. These local acceleration/deceleration of the fluids could be significant in the interface between the proppants in the fracture and formation rock. It has been proved that the non-Darcy factor is not upscalable [13]. This work attempts to demonstrate the local nature of non-Darcy flows through numerical simulations.

In this work, numerical flow simulations are run for simple geometries of 2 dimensional circle packs and also on the combination of the circle packs with different circle sizes. Figure 4.1 shows a combination of two circle packs with two different circle sizes. 


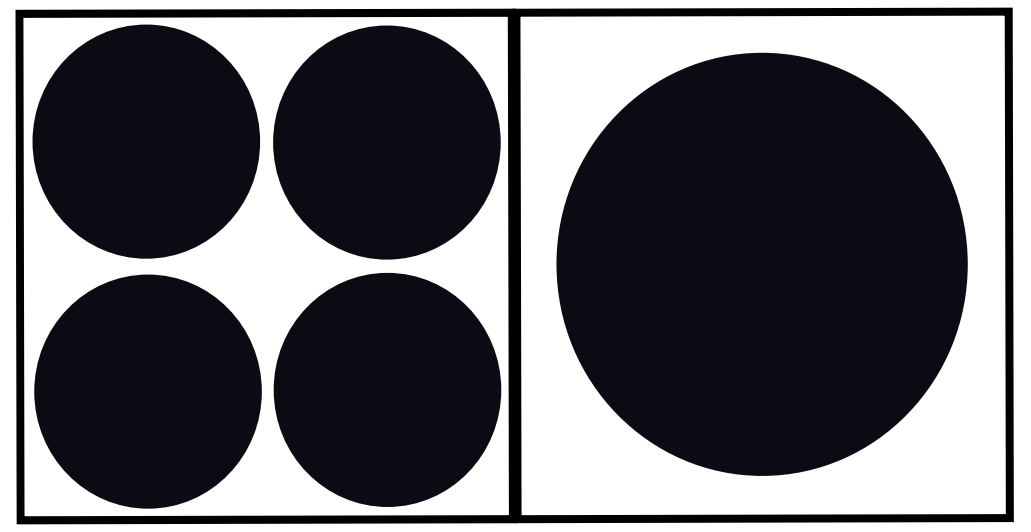

Figure 4.1: Representation of combination of two circle packs with different circle sizes connected with each other to make a combined geometry $(1 \times 1)$ and $(2 \times 2)$ circle pack

Circle packs with a single circle size and combined circle packs are simulated to predict permeability and the non-Darcy factor.

Each simulation starts with a very low body force and therefore (low Reynolds/Forchheimer number) so that the value of the permeability can be predicted. The value of the body force is gradually increased in each new simulation until a noticeable reduction in the apparent permeability is observed. The value of the body force is increased, until the simulation breaks down when (incompressibility assumption is no longer valid). Subsequently, apparent permeability is then plotted against the Forchheimer number, $F_{o}$, in a log-log plot to observe the reduction of the apparent permeability values.

To predict the value of the non-Darcy factor, first, the apparent permeability is calculated for each value of body force via equation (1.3); subsequently, the values of the apparent permeability are converted from lattice units to physical units. The converted apparent permeabilities are plotted against $\frac{u}{\nu}$. The slope of the line is the non-Darcy factor.

\subsection{Numerical Simulation Results}

In this section, LBM simulation results are performed. For each circle pack and combined circle packs simulations for velocity streamlines are presented to show the behavior of the fluid flow under low and high fluid velocities (low and high Reynolds/Forchheimer number). 
Figure 4.2.a shows the plot of the apparent permeability against the Forchheimer numbers. It shows that there is a reduction of apparent permeability from $1.88 \times 10^{-6} \mathrm{~m}^{2}$ at $F_{o}=9.97 \times 10^{-5}$ to $1.51 \times 10^{-6} m^{2}$ at $F_{o}=1.80 \times 10^{-1}$ with $(19.81$ percent reduction $)$. Figure 4.2.b shows a linear relationship between the inverse of apparent permeability and velocity over kinematic viscosity, $\frac{u}{\nu}$ with $R^{2}=0.9999$ and a non-Darcy factor of $14.765 \mathrm{~m}^{-1}$.

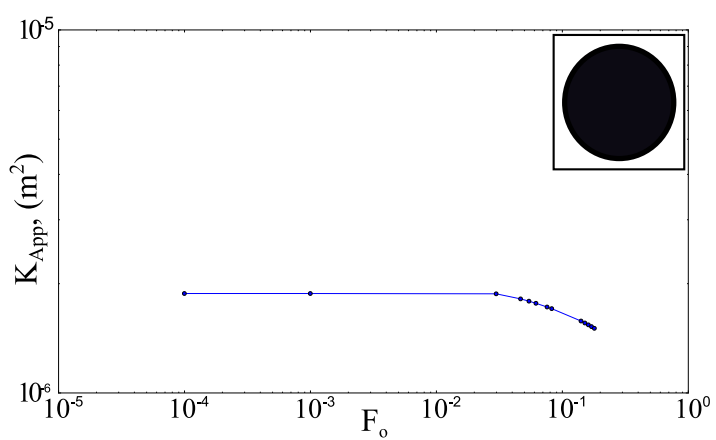

(a) Apparent permeability versus Forchheimer number

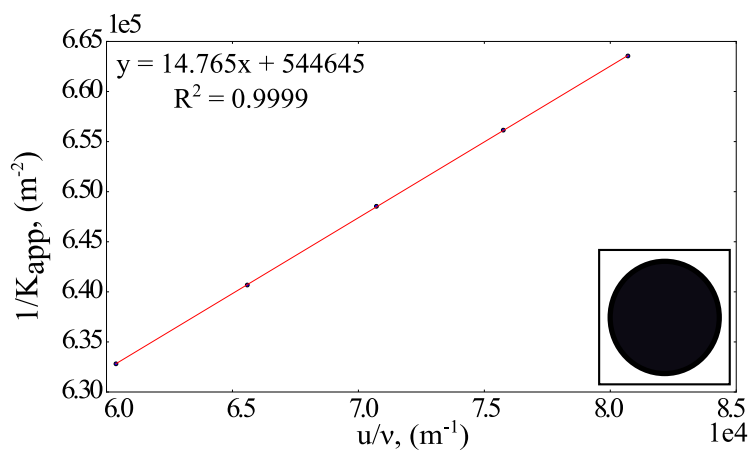

(b) Inverse apparent permeability versus $\mathrm{u} / \nu$

Figure 4.2: Simulations results for a single circle

Figures 4.3 show that the velocity streamlines for a single circle at $F_{o}=9.97 \times 10^{-5}$ Figure 4.3.a and $F_{o}=1.80 \times 10^{-1}$ Figure 4.3.b. Due to the acceleration/deceleration of the fluid flow, at high $F_{o}$ some eddies are generated in Figure 4.3.b. 

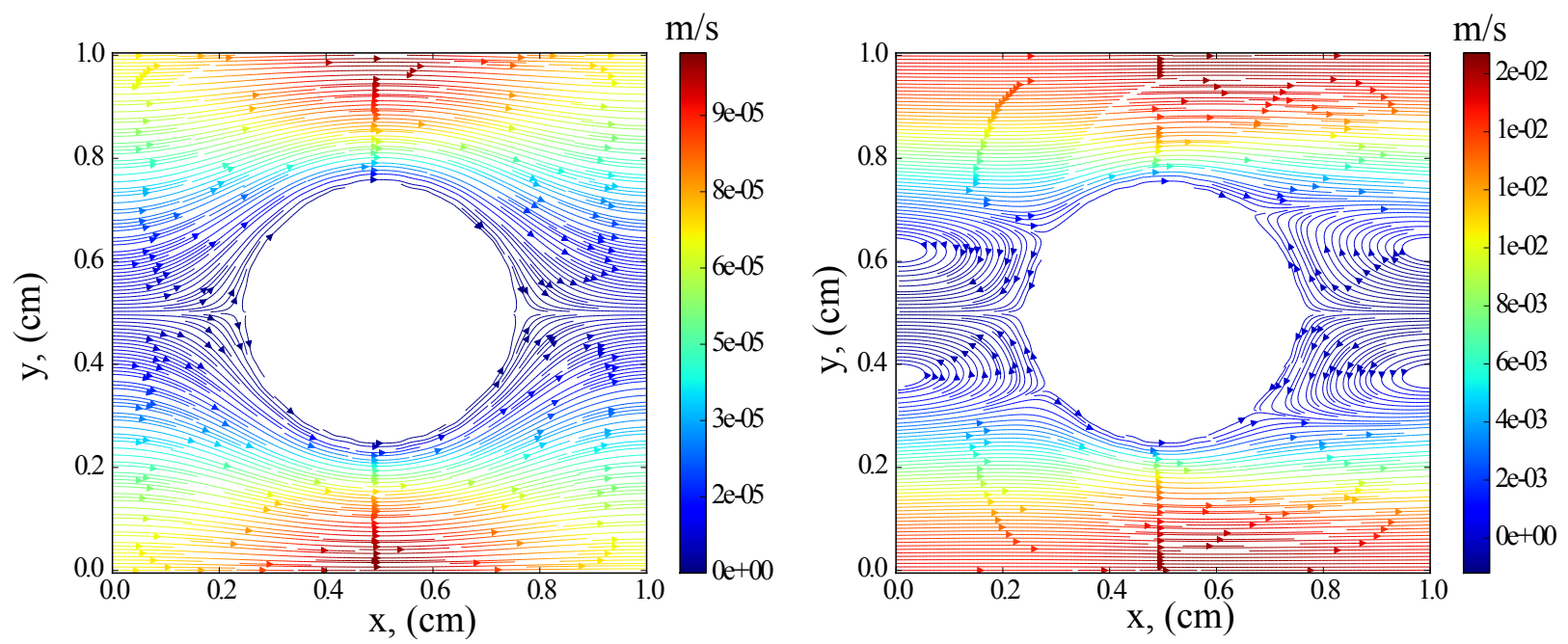

(a) Velocity streamlines for a single circle

(b) Velocity streamlines for a single circle with $F_{o}=9.97 \times 10^{-5}$ with $F_{o}=1.80 \times 10^{-1}$

Figure 4.3: Velocity streamlines for a single circle $F_{o}=9.97 \times 10^{-5}$ (left) and $F_{o}=1.80 \times 10^{-1}$ (right)

Figure 4.4.a shows the plot of the apparent permeability against the Forchheimer numbers. It shows that there is a reduction of apparent permeability from $4.82 \times 10^{-7} \mathrm{~m}^{2}$ at $F_{o}=3.09 \times 10^{-3}$ to $4.00 \times 10^{-7} \mathrm{~m}^{2}$ at $F_{o}=2.12 \times 10^{-1}$ with (17.09 percent reduction) Figure 4.4.b shows a linear relationship between the inverse of apparent permeability and velocity over kinematic viscosity, $\frac{u}{\nu}$ with $R^{2}=1$ and a non-Darcy factor of $69.427 \mathrm{~m}^{-1}$.

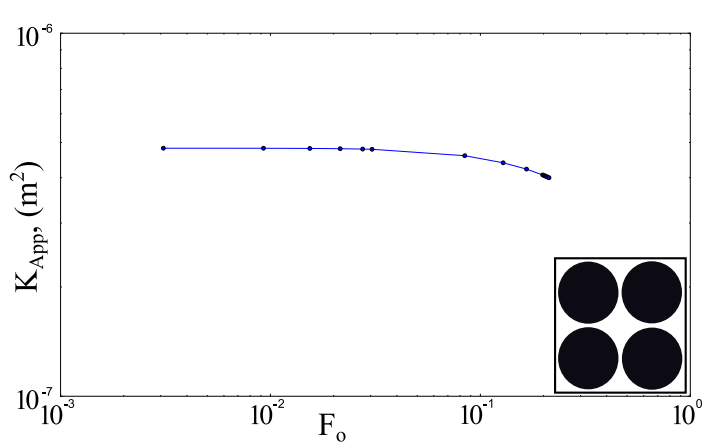

(a) Apparent permeability versus Forchheimer number

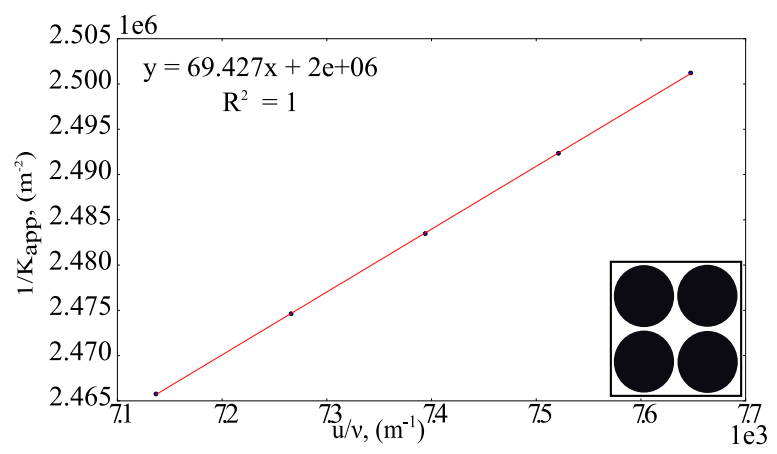

(b) Inverse apparent permeability versus $\mathrm{u} / \nu$

Figure 4.4: Simulations results for $(2 \times 2)$ circle pack

Figure 4.5 show that the velocity streamlines for $(2 \times 2)$ circle pack at $F_{o}=3.09 \times 10^{-3}$ 
Figure 4.3.a and $F_{o}=2.12 \times 10^{-1}$ Figure 4.5.b. Due to the acceleration/deceleration of the fluid flow, at high $F_{o}$ some eddies are generated in Figure 4.5.b.

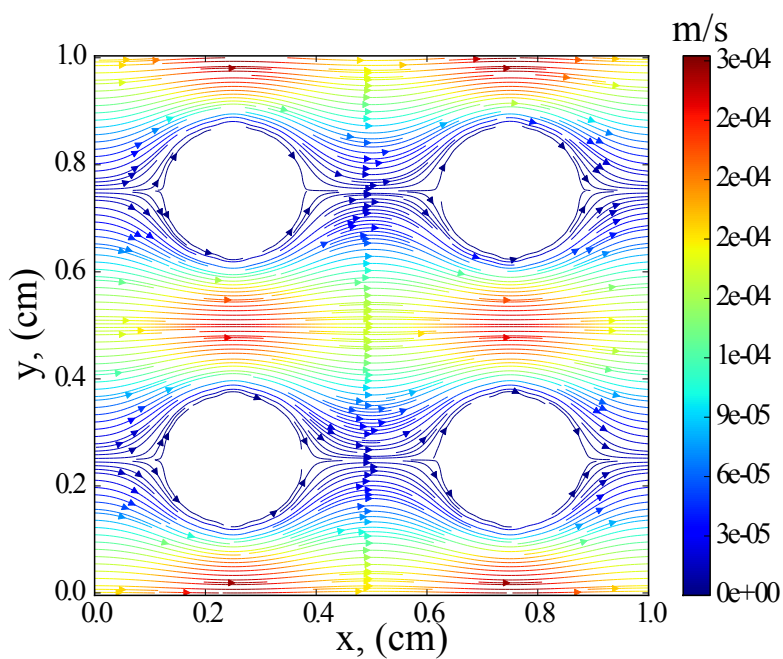

(a) Velocity streamlines for $(2 \times 2)$ circle pack with $F_{o}=3.09 \times 10^{-3}$

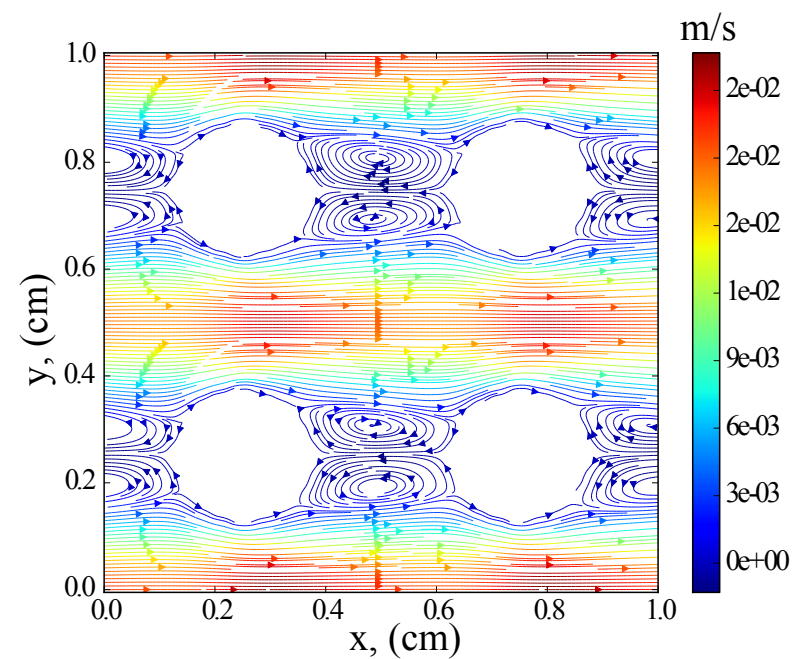

(b) Velocity streamlines for $(2 \times 2)$ circle pack with $F_{o}=2.12 \times 10^{-1}$

Figure 4.5: Velocity streamlines for $(2 \times 2)$ circle pack $F_{o}=3.09 \times 10^{-3}$ (left) and $F_{o}=$ $2.12 \times 10^{-1}$ (right)

Figure 4.6.a shows the plot of the apparent permeability against the Forchheimer numbers. It shows that there is a reduction of apparent permeability from $2.18 \times 10^{-7} \mathrm{~m}^{2}$ at $F_{o}=1.24 \times 10^{-5}$ to $1.84 \times 10^{-7} m^{2}$ at $F_{o}=2.29 \times 10^{-1}$ with (15.69 percent reduction) Figure 4.6.b shows a linear relationship between the inverse of apparent permeability and velocity over kinematic viscosity, $\frac{u}{\nu}$ with $R^{2}=0.9995$ and a non-Darcy factor of $136.03 \mathrm{~m}^{-1}$.

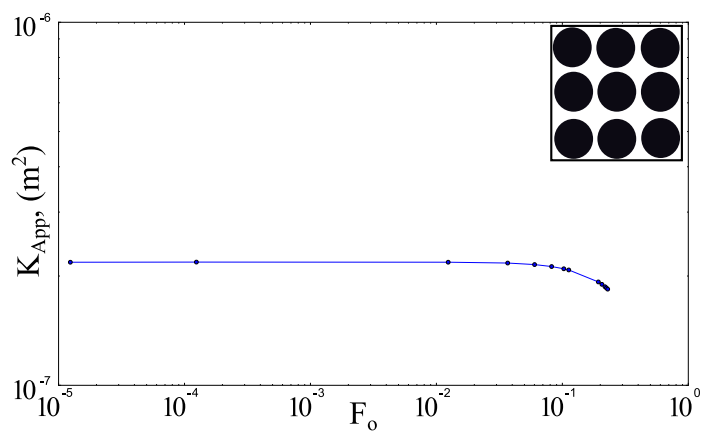

(a) Apparent permeability versus Forchheimer number

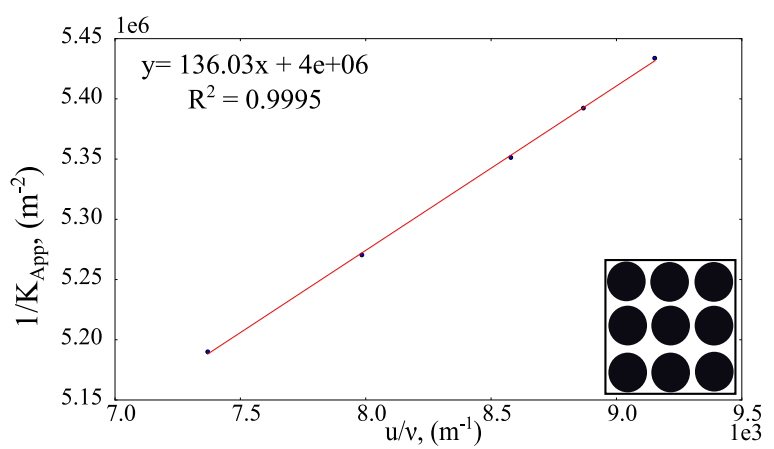

(b) Inverse apparent permeability versus $\mathrm{u} / \nu$

Figure 4.6: Simulations results for $(3 \times 3)$ circle pack 
Figure 4.7 show that the velocity streamlines for $(3 \times 3)$ circle pack at $F_{o}=1.24 \times 10^{-5}$ Figure 4.7.a and $F_{o}=2.29 \times 10^{-1}$ Figure 4.7.b. Due to the acceleration/deceleration of the fluid flow, at high $F_{o}$ some eddies are generated in Figure 4.7.b.
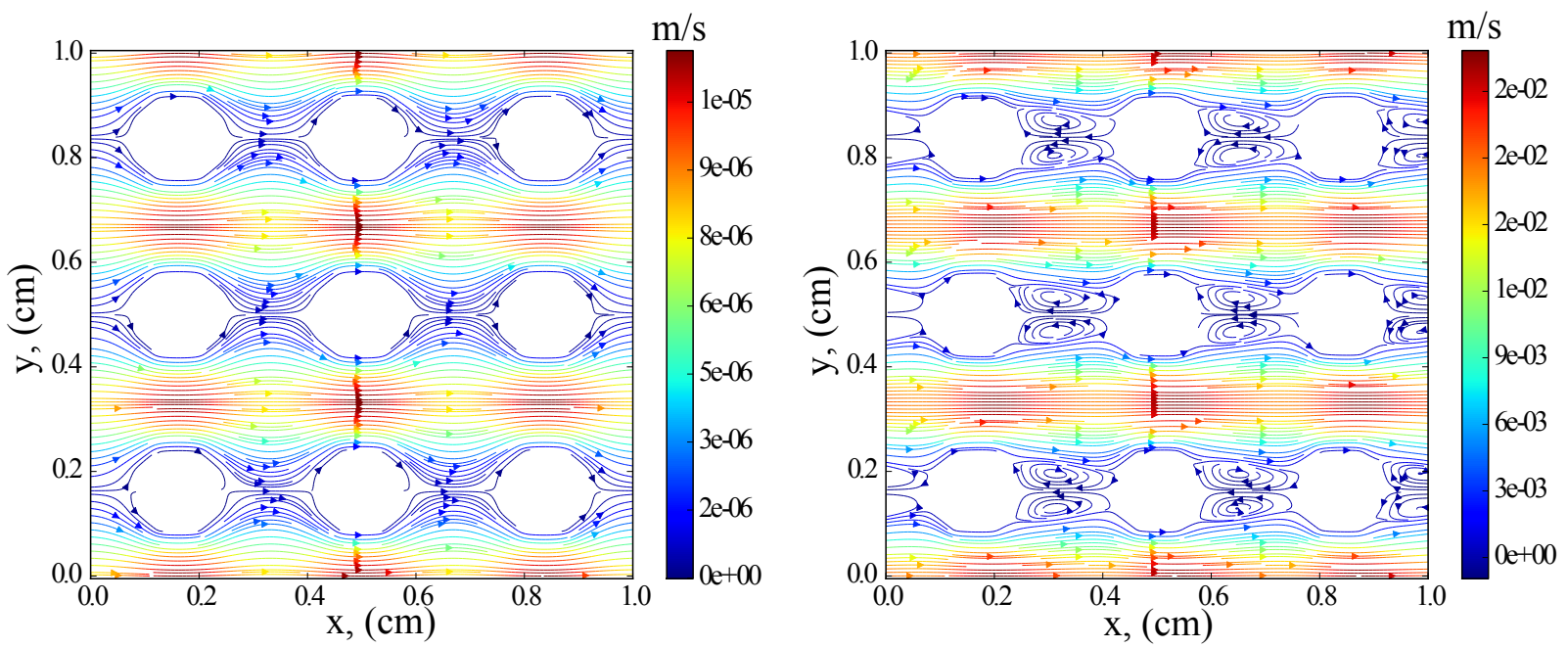

(a) Velocity streamlines for $(3 \times 3)$ circle pack with $F_{o}=1.24 \times 10^{-5}$

(b) Velocity streamlines for $(3 \times 3)$ circle pack with $F_{o}=2.29 \times 10^{-1}$

Figure 4.7: Velocity streamlines for $(3 \times 3)$ circle pack $F_{o}=1.24 \times 10^{-5}$ (left) and $F_{o}=$ $2.29 \times 10^{-1}$ (right)

Figure 4.8.a shows the plot of the apparent permeability against the Forchheimer numbers. It shows that there is a reduction of apparent permeability from $1.19 \times 10^{-7} \mathrm{~m}^{2}$ at $F_{o}=8.23 \times 10^{-4}$ to $1.03 \times 10^{-7} \mathrm{~m}^{2}$ at $F_{o}=2.70 \times 10^{-1}$ with $(13.71$ percent reduction) . Figure 4.8.b shows a linear relationship between the inverse of apparent permeability and velocity over kinematic viscosity, $\frac{u}{\nu}$ with $R^{2}=0.9996$ and a non-Darcy factor of $302.73 \mathrm{~m}^{-1}$. 


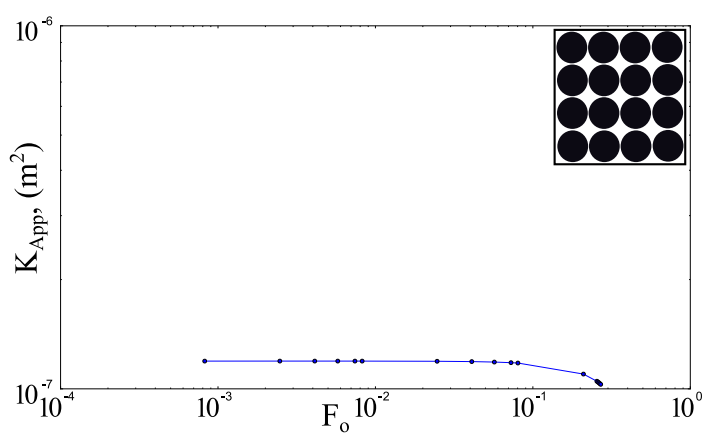

(a) Apparent permeability versus Forchheimer number

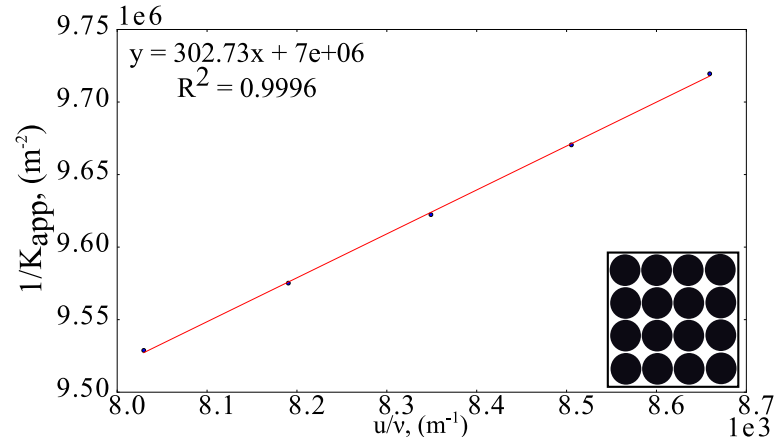

(b) Inverse apparent permeability versus $\mathrm{u} / \nu$

Figure 4.8: Simulations results for $(4 \times 4)$ circle pack

Figure 4.9 show that the velocity streamlines for $(4 \times 4)$ circle pack at $F_{o}=8.23 \times 10^{-4}$ Figure 4.9.a and $F_{o}=2.70 \times 10^{-1}$ Figure 4.9.b. Due to the acceleration/deceleration of the fluid flow, at high $F_{o}$ some eddies are generated in Figure 4.9.b.

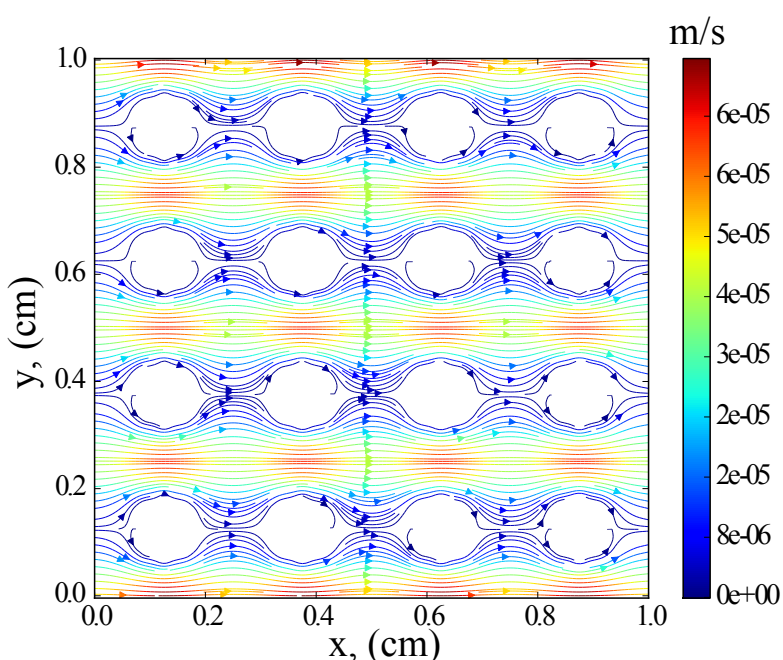

(a) Velocity streamlines $(4 \times 4)$ circle pack with $F_{o}=8.23 \times 10^{-4}$

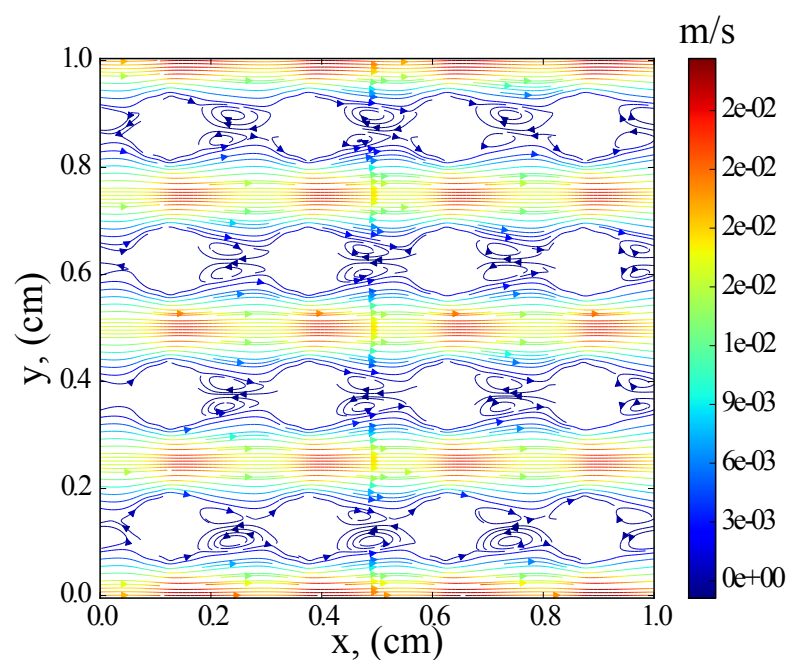

(b) Velocity streamlines for $(4 \times 4)$ circle pack with $F_{o}=2.70 \times 10^{-1}$

Figure 4.9: Velocity streamlines for $(4 \times 4)$ circle pack $F_{o}=8.23 \times 10^{-4}$ (left) and $F_{o}=$ $2.70 \times 10^{-1}$ (right)

Figure 4.10.a shows the plot of the apparent permeability against the Forchheimer numbers. It shows that there is a reduction of apparent permeability from $1.87 \times 10^{-7} \mathrm{~m}^{2}$ at $F_{o}=1.24 \times 10^{-3}$ to $1.26 \times 10^{-7} m^{2}$ at $F_{o}=4.43 \times 10^{-1}$ with $(32.27$ percent reduction $)$. 
Figure 4.10.b shows a linear relationship between the inverse of apparent permeability and velocity over kinematic viscosity, $\frac{u}{\nu}$ with $R^{2}=1$ and a non-Darcy factor of $232.32 \mathrm{~m}^{-1}$.

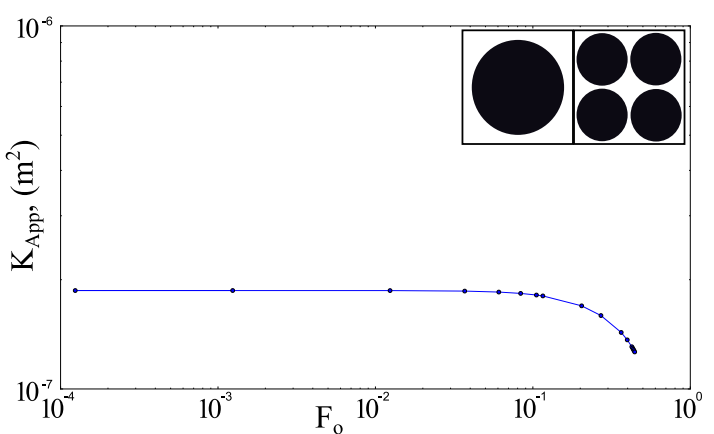

(a) Apparent permeability versus Forchheimer number

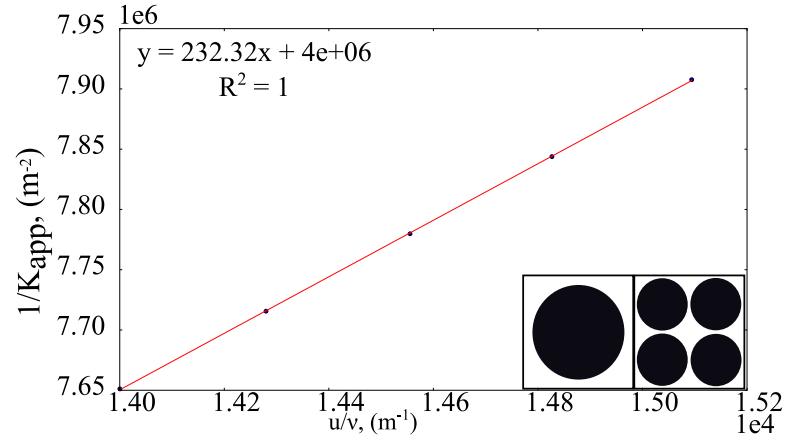

(b) Inverse apparent permeability versus $\mathrm{u} / \nu$

Figure 4.10: Simulations results for $(1 \times 1)+(2 \times 2)$ circle pack

Figure 4.11.a shows that the velocity streamlines for $(1 \times 1)+(2 \times 2)$ circle pack at $F_{o}=1.24 \times 10^{-3}$ are smooth and parallel. Figure 4.11.b shows some eddies in velocity streamline plot for $F_{o}=4.43 \times 10^{-1}$ due to the acceleration/deceleration of the fluid flow. Eddies are generated mostly in the interface between single circle and $(2 \times 2)$ circle pack.

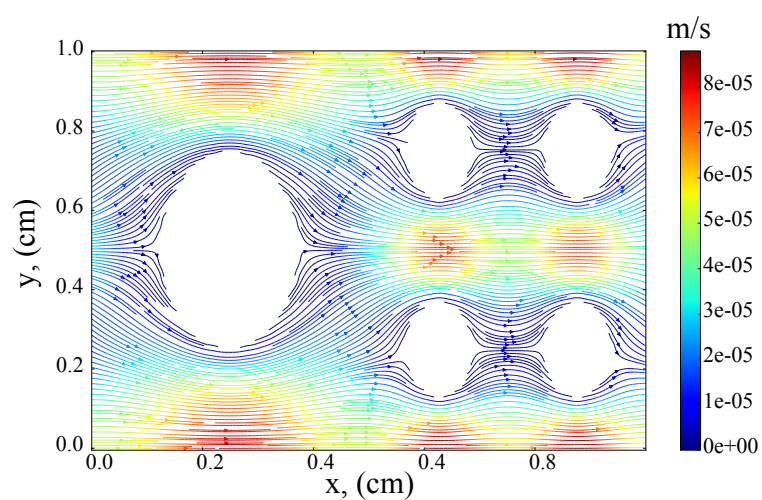

(a) Velocity streamlines for $(1 \times 1)+(2 \times 2)$ circle pack with $F_{o}=1.24 \times 10^{-3}$

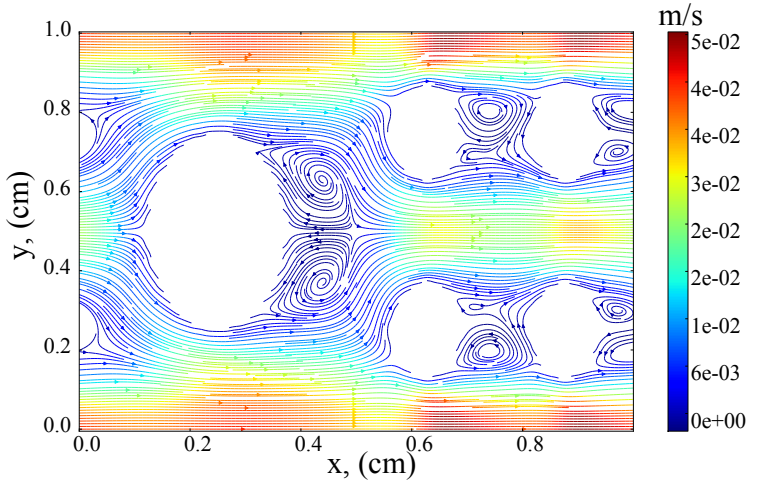

(b) Velocity streamlines for $(1 \times 1)+(2 \times 2)$ circle pack with $F_{o}=4.43 \times 10^{-1}$

Figure 4.11: Velocity streamlines for $(1 \times 1)+(2 \times 2)$ circle pack $F_{o}=1.24 \times 10^{-3}$ (left) and $F_{o}=4.43 \times 10^{-1}$ (right)

Figure 4.12.a shows the plot of the apparent permeability against the Forchheimer numbers. It shows that there is a reduction of apparent permeability from $9.56 \times 10^{-8} \mathrm{~m}^{2}$ at 
$F_{o}=4.83 \times 10^{-5}$ to $7.11 \times 10^{-8} m^{2}$ at $F_{o}=3.74 \times 10^{-1}$ with $(25.62$ percent reduction $)$. Figure 4.12.b shows a linear relationship between the inverse of apparent permeability and velocity over kinematic viscosity, $\frac{u}{\nu}$ with $R^{2}=0.9991$ and a non-Darcy factor of $345.75 \mathrm{~m}^{-1}$.

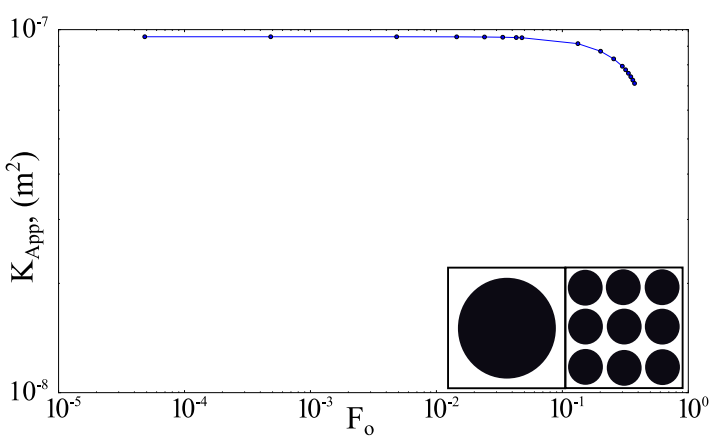

(a) Apparent permeability versus Forchheimer number

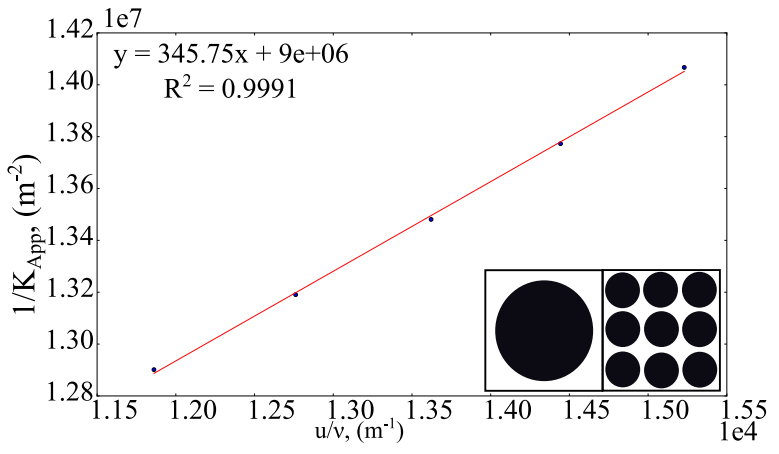

(b) Inverse apparent permeability versus $\mathrm{u} / \nu$

Figure 4.12: Simulations results for $(1 \times 1)+(3 \times 3)$ circle pack

Figure 4.13.a shows that the velocity streamlines for $(1 \times 1)+(3 \times 3)$ circle pack at $F_{o}=4.83 \times 10^{-5}$ are smooth and parallel. Figure 4.13.b shows some eddies in velocity streamline plot for $F_{o}=3.74 \times 10^{-1}$ due to the acceleration/deceleration of the fluid flow. Eddies are generated mostly in the interface between single circle and $(3 \times 3)$ circle pack.

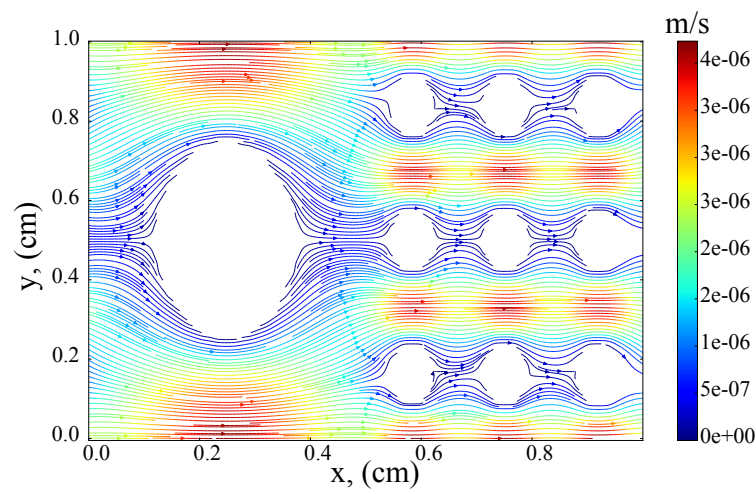

(a) Velocity streamlines for $(1 \times 1)+(3 \times 3)$ circle pack with $F_{o}=4.83 \times 10^{-5}$

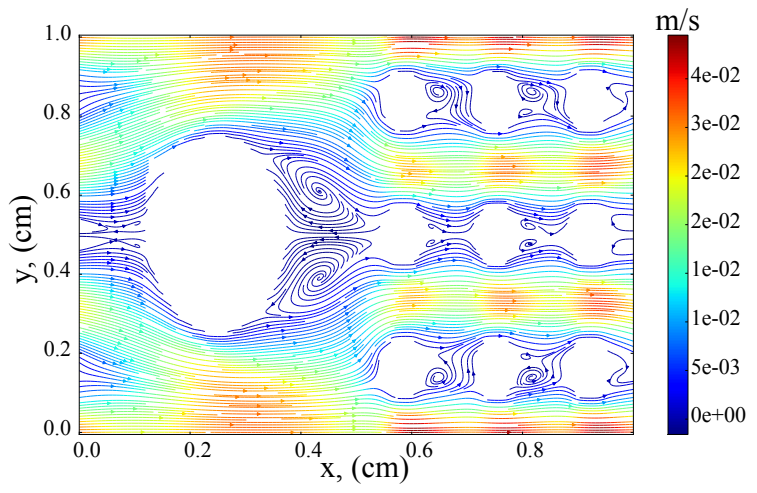

(b) Velocity streamlines for a $(1 \times 1)+(3 \times 3)$ circle pack with $F_{o}=3.74 \times 10^{-1}$

Figure 4.13: Velocity streamlines for $(1 \times 1)+(3 \times 3)$ circle pack $F_{o}=4.83 \times 10^{-5}$ (left) and $F_{o}=3.74 \times 10^{-1}$ (right)

Figure 4.14.a shows the plot of the apparent permeability against the Forchheimer num- 
bers. It shows that there is a reduction of apparent permeability from $5.54 \times 10^{-8} \mathrm{~m}^{2}$ at $F_{o}=1.85 \times 10^{-3}$ to $4.58 \times 10^{-8} \mathrm{~m}^{2}$ at $F_{o}=2.53 \times 10^{-1}$ with (17.27 percent reduction). Figure 4.14.b shows a linear relationship between the inverse of apparent permeability and velocity over kinematic viscosity, $\frac{u}{\nu}$ with $R^{2}=0.9928$ and a non-Darcy factor of $393.09 \mathrm{~m}^{-1}$.

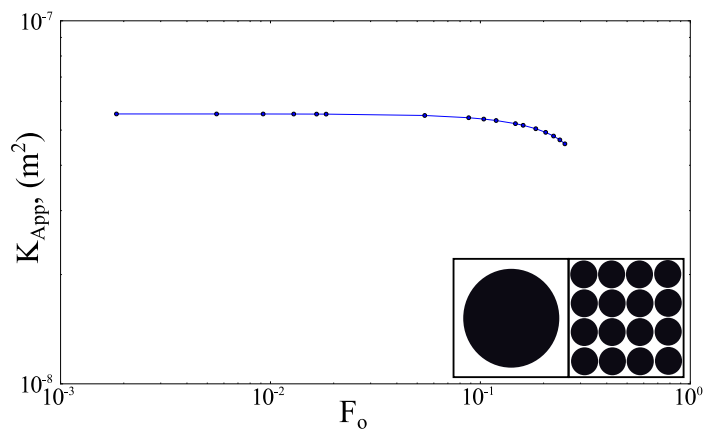

(a) Apparent permeability versus Forchheimer number

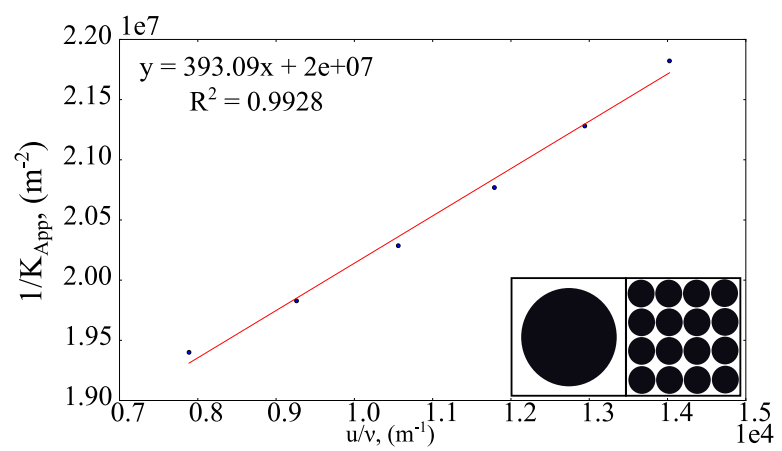

(b) Inverse apparent permeability versus $\mathrm{u} / \nu$

Figure 4.14: Simulations results for $(1 \times 1)+(4 \times 4)$ circle pack

Figure 4.15.a shows that the velocity streamlines for $(1 \times 1)+(4 \times 4)$ circle pack at $F_{o}=1.85 \times 10^{-3}$ are smooth and parallel. Figure 4.15.b shows some eddies in velocity streamline plot for $F_{o}=2.53 \times 10^{-1}$ due to the acceleration/deceleration of the fluid flow. Eddies are generated mostly in the interface between single circle and $(4 \times 4)$ circle pack.

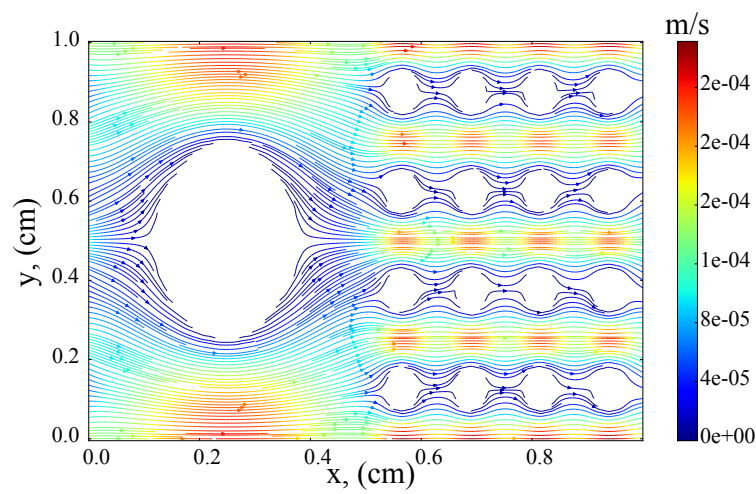

(a) Velocity streamlines for $(1 \times 1)+(4 \times 4)$ circle pack with $F_{o}=1.85 \times 10^{-3}$

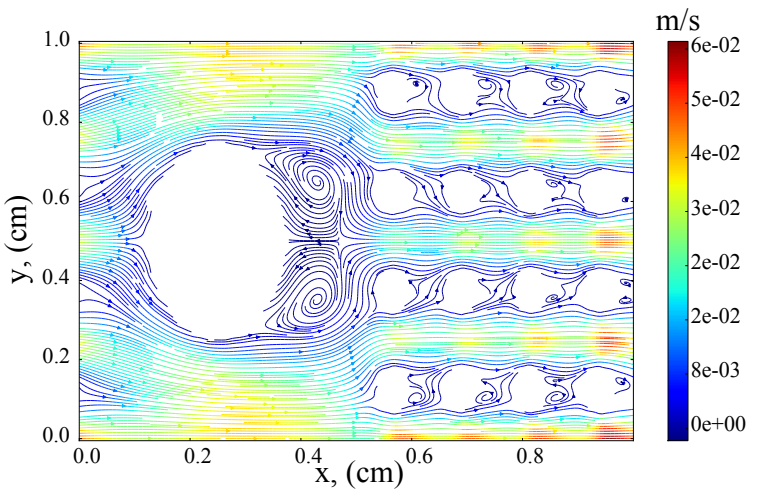

(b) Velocity streamlines for $(1 \times 1)+(4 \times 4)$ circle pack with $F_{o}=2.53 \times 10^{-1}$

Figure 4.15: Velocity streamlines for $(1 \times 1)+(4 \times 4)$ circle pack $F_{o}=1.85 \times 10^{-3}$ (left) and $F_{o}=2.53 \times 10^{-1}$ (right) 
Figure 4.16.a shows the plot of the apparent permeability against the Forchheimer numbers. It shows that there is a reduction of apparent permeability from $7.34 \times 10^{-8} \mathrm{~m}^{2}$ at $F_{o}=2.62 \times 10^{-5}$ to $5.76 \times 10^{-8} \mathrm{~m}^{2}$ at $F_{o}=2.90 \times 10^{-1}$ with $(21.59$ percent reduction $)$. Figure 4.16.b shows a linear relationship between the inverse of apparent permeability and velocity over kinematic viscosity, $\frac{u}{\nu}$ with $R^{2}=0.9993$ and a non-Darcy factor of $317.9 \mathrm{~m}^{-1}$.

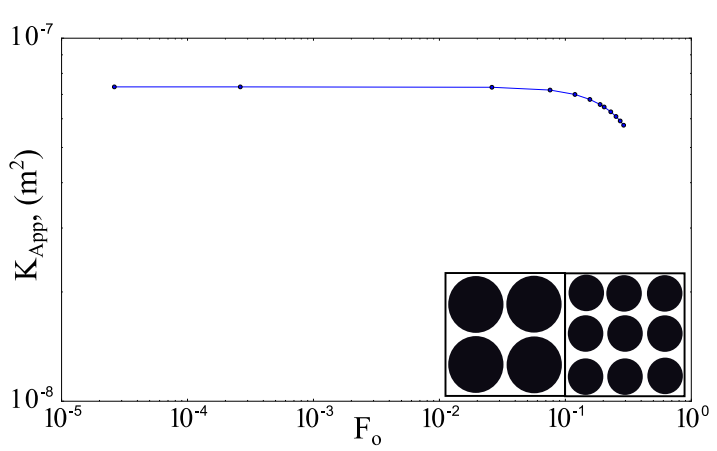

(a) Apparent permeability versus Forchheimer number

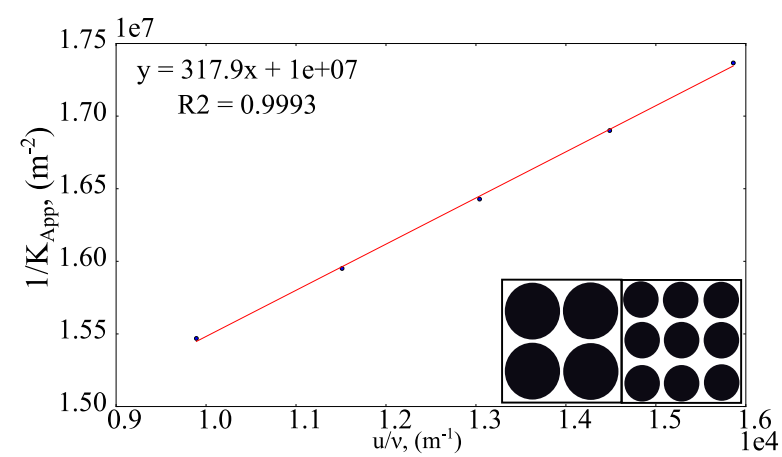

(b) Inverse apparent permeability versus $\mathrm{u} / \nu$

Figure 4.16: Simulations results for $(2 \times 2)+(3 \times 3)$ circle pack

Figure 4.17.a shows that the velocity streamlines for $(2 \times 2)+(3 \times 3)$ circle pack at $F_{o}=2.62 \times 10^{-5}$ are smooth and parallel. Figure 4.17.b shows some eddies in velocity streamline plot for $\left(F_{o}=2.90 \times 10^{-1}\right.$ due to the acceleration/deceleration of the fluid flow. Eddies are generated mostly in the interface between $(2 \times 2)+(3 \times 3)$ circle pack.

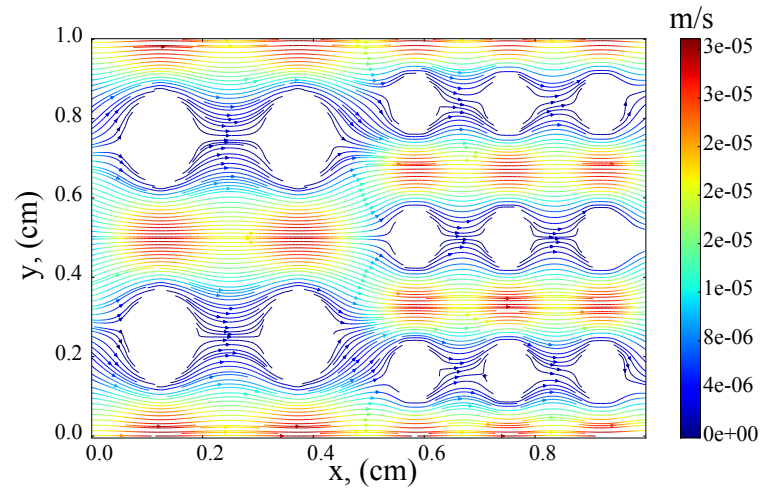

(a) Velocity streamlines for $(2 \times 2)+(3 \times 3)$ circle pack with $F_{o}=2.62 \times 10^{-5}$

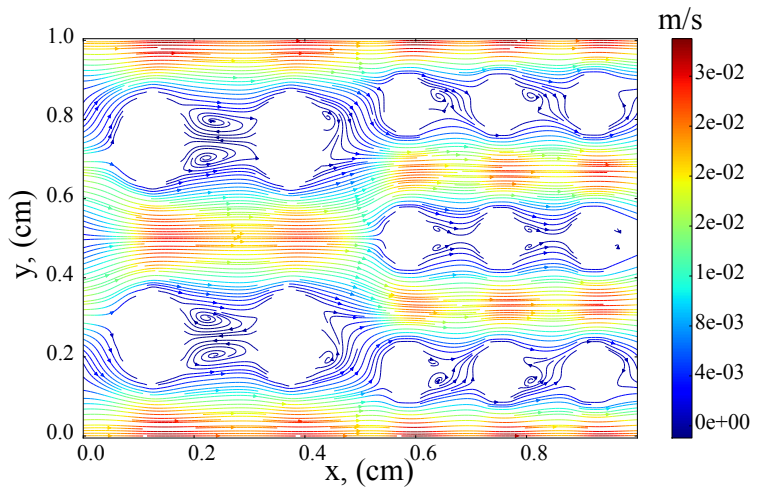

(b) Velocity streamlines for $(2 \times 2)+(3 \times 3)$ circle pack with $F_{o}=2.90 \times 10^{-1}$

Figure 4.17: Velocity streamlines for $(2 \times 2)+(3 \times 3)$ circle pack $F_{o}=2.62 \times 10^{-5}$ (left) and $F_{o}=2.90 \times 10^{-1}$ (right) 
Figure 4.18.a shows the plot of the apparent permeability against the Forchheimer numbers. It shows that there is a reduction of apparent permeability from $4.71 \times 10^{-8} \mathrm{~m}^{2}$ at $F_{o}=1.29 \times 10^{-5}$ to $4.02 \times 10^{-8} m^{2}$ at $F_{o}=2.25 \times 10^{-1}$ with (14.67 percent reduction) . Figure 4.18.b shows a linear relationship between the inverse of apparent permeability and velocity over kinematic viscosity, $\frac{u}{\nu}$ with $R^{2}=1$ and a non-Darcy factor of $379.12 \mathrm{~m}^{-1}$.

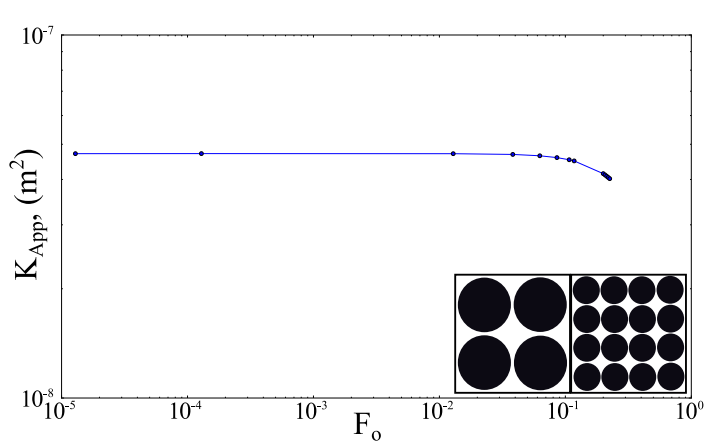

(a) Apparent permeability versus Forchheimer number

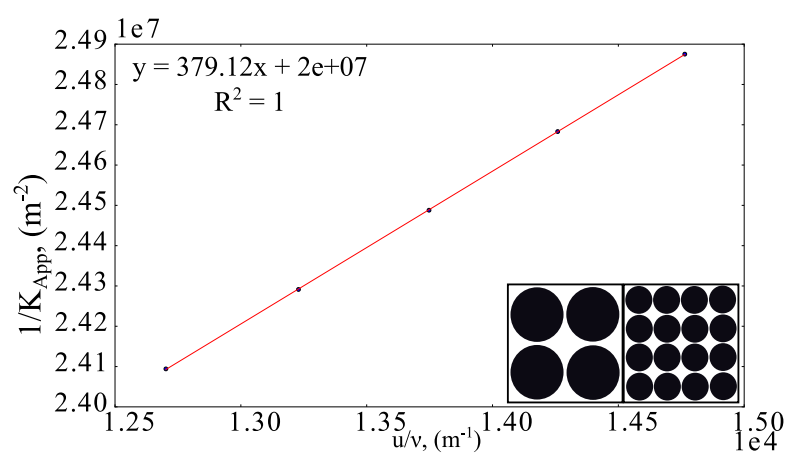

(b) Inverse apparent permeability versus $\mathrm{u} / \nu$

Figure 4.18: Simulations results for $(2 \times 2)+(4 \times 4)$ circle pack

Figure 4.19.a shows that the velocity streamlines for $(2 \times 2)+(4 \times 4)$ circle pack at $F_{o}=1.29 \times 10^{-5}$ are smooth and parallel. Figure 4.19.b shows some eddies in velocity streamline plot for $F_{o}=2.25 \times 10^{-1}$ due to the acceleration/deceleration of the fluid flow. Eddies are generated mostly in the interface between $(2 \times 2)+(4 \times 4)$ circle pack.

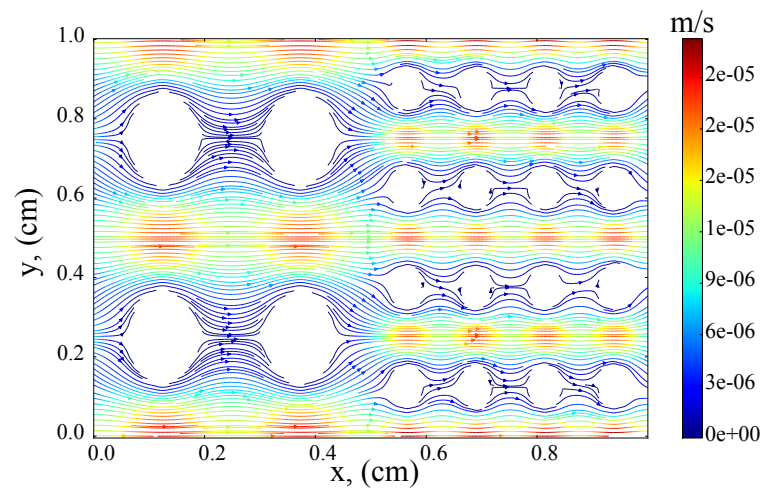

(a) Velocity streamlines for $(2 \times 2)+(4 \times 4)$ circle pack with $F_{o}=1.29 \times 10^{-5}$

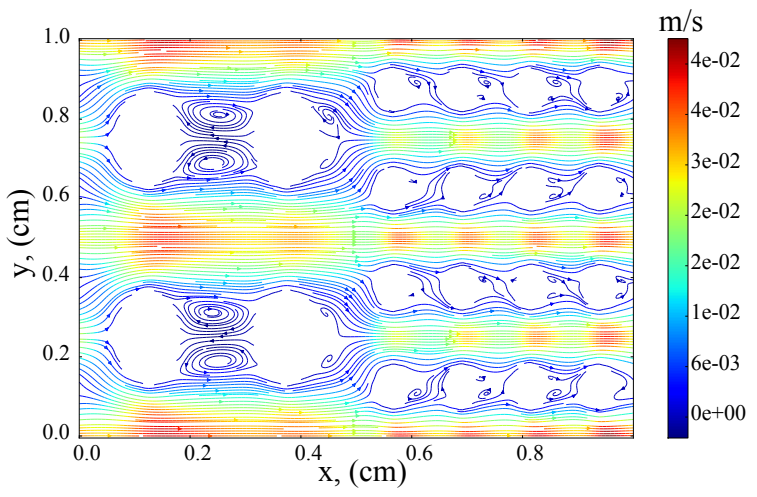

(b) Velocity streamlines for $(2 \times 2)+(4 \times 4)$ circle pack with $F_{o}=2.25 \times 10^{-1}$

Figure 4.19: Velocity streamlines for $(2 \times 2)+(4 \times 4)$ circle pack $F_{o}=1.29 \times 10^{-5}$ (left) and $F_{o}=2.25 \times 10^{-1}$ (right) 
Figure 4.20.a shows the plot of the apparent permeability against the Forchheimer numbers. It shows that there is a reduction of apparent permeability from $3.80 \times 10^{-8} \mathrm{~m}^{2}$ at $F_{o}=9.75 \times 10^{-6}$ to $3.24 \times 10^{-8} m^{2}$ at $F_{o}=2.27 \times 10^{-1}$ with (14.69 percent reduction) . Figure 4.20.b shows a linear relationship between the inverse of apparent permeability and velocity over kinematic viscosity, $\frac{u}{\nu}$ with $R^{2}=0.9998$ and a non-Darcy factor of $440.44 \mathrm{~m}^{-1}$.

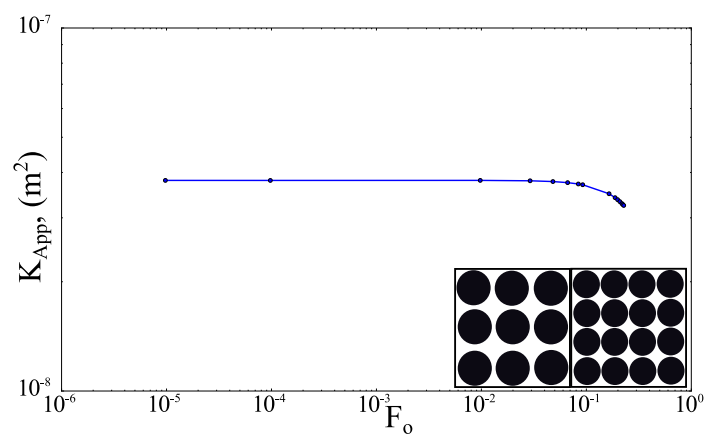

(a) Apparent permeability versus Forchheimer number

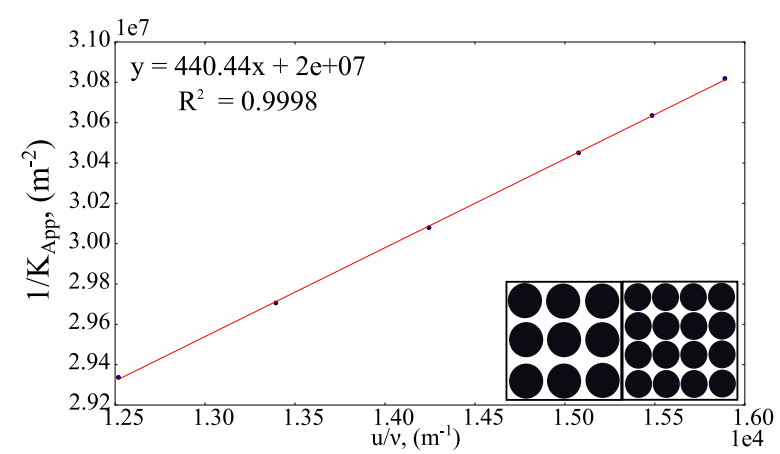

(b) Inverse apparent permeability versus $\mathrm{u} / \nu$

Figure 4.20: Simulations results for $(3 \times 3)+(4 \times 4)$ circle pack

Figure 4.21.a shows that the velocity streamlines for $(3 \times 3)+(4 \times 4)$ circle pack at $F_{o}=9.75 \times 10^{-6}$ are smooth and parallel. Figure 4.21.b shows some eddies in velocity streamline plot for $F_{o}=2.27 \times 10^{-1}$ due to the acceleration/deceleration of the fluid flow. Eddies are generated mostly in the interface between $(3 \times 3)+(4 \times 4)$ circle pack.

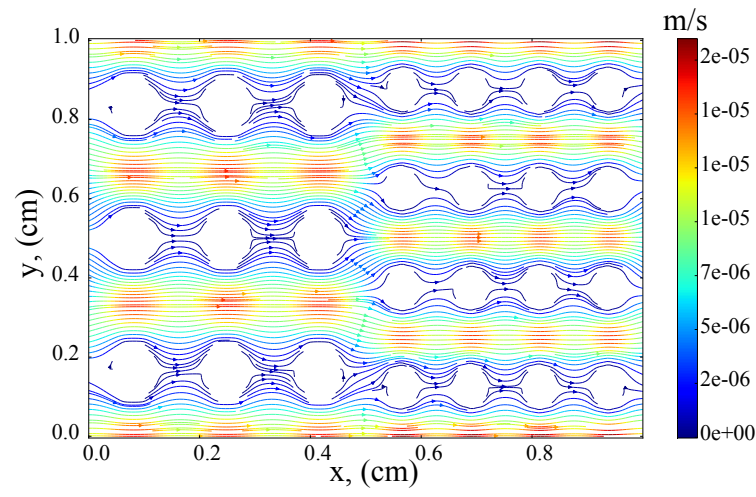

(a) Velocity streamlines for $(3 \times 3)+(4 \times 4)$ circle pack with $F_{o}=9.75 \times 10^{-6}$

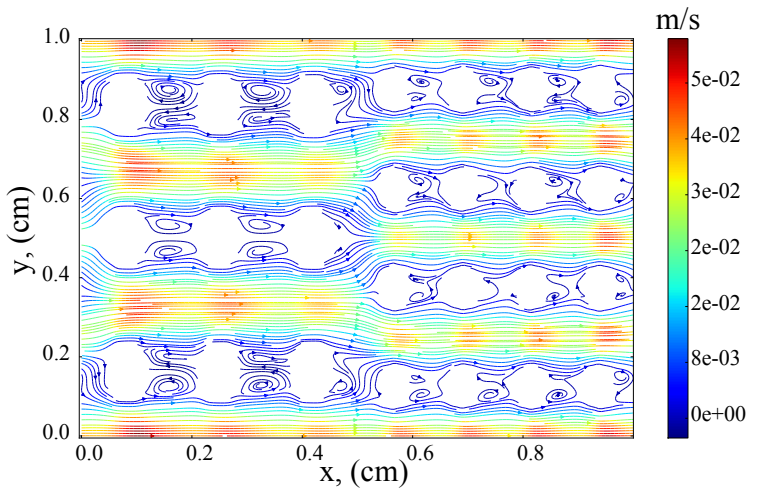

(b) Velocity streamlines for $(3 \times 3)+(4 \times 4)$ circle pack with $F_{o}=2.27 \times 10^{-1}$

Figure 4.21: Velocity streamlines for $(3 \times 3)+(4 \times 4)$ circle pack $F_{o}=9.75 \times 10^{-6}$ (left) and $F_{o}=2.27 \times 10^{-1}$ (right) 
Figure 4.22.a shows the plot of the apparent permeability against the Forchheimer numbers. It shows that there is a reduction of apparent permeability from $2.58 \times 10^{-8} \mathrm{~m}^{2}$ at $F_{o}=4.87 \times 10^{-3}$ to $2.31 \times 10^{-8} m^{2}$ at $F_{o}=1.96 \times 10^{-1}$ with $(10.19$ percent reduction $)$. Figure 4.22.b shows a linear relationship between the inverse of apparent permeability and velocity over kinematic viscosity, $\frac{u}{\nu}$ with $R^{2}=0.9994$ and a non-Darcy factor of $3981 \mathrm{~m}^{-1}$.

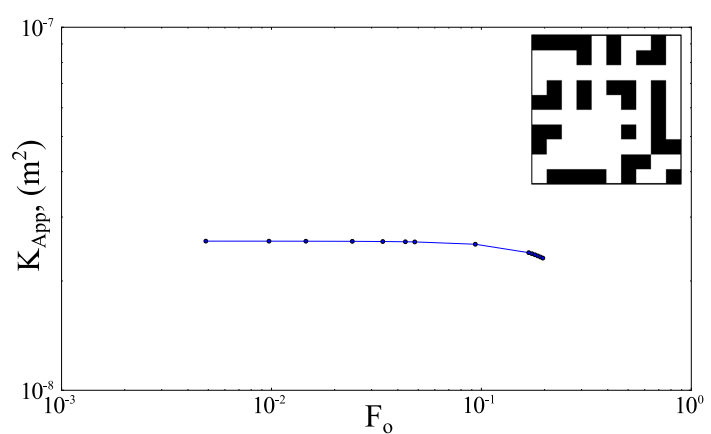

(a) Apparent permeability versus Forchheimer

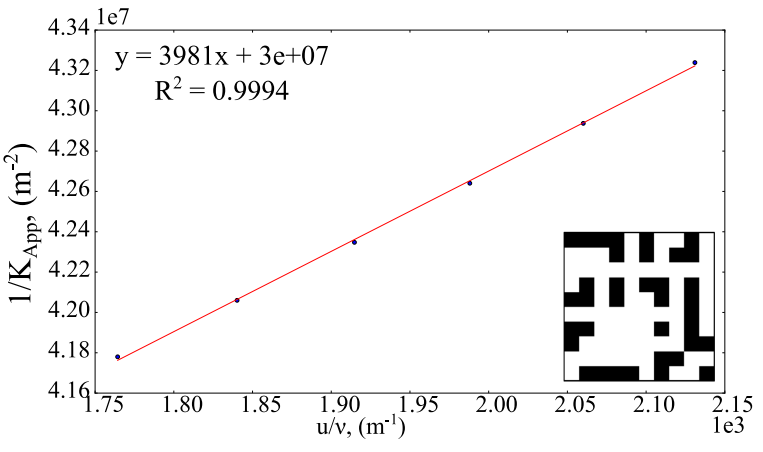

(b) Inverse apparent permeability versus $\mathrm{u} / \nu$ number for random porous media with 0.64 porosity for random porous media with 0.64 porosity

Figure 4.22: Simulations results of 2-dimensional random porous media for 0.64 porosity

Figure 4.23.a shows that the velocity streamlines for porosity (0.64) random 2D porous media at $F_{o}=4.87 \times 10^{-3}$ are smooth and parallel. Figure 4.23.b shows some eddies in velocity streamline plot for $F_{o}=1.96 \times 10^{-1}$ due to the acceleration/deceleration of the fluid flow. 

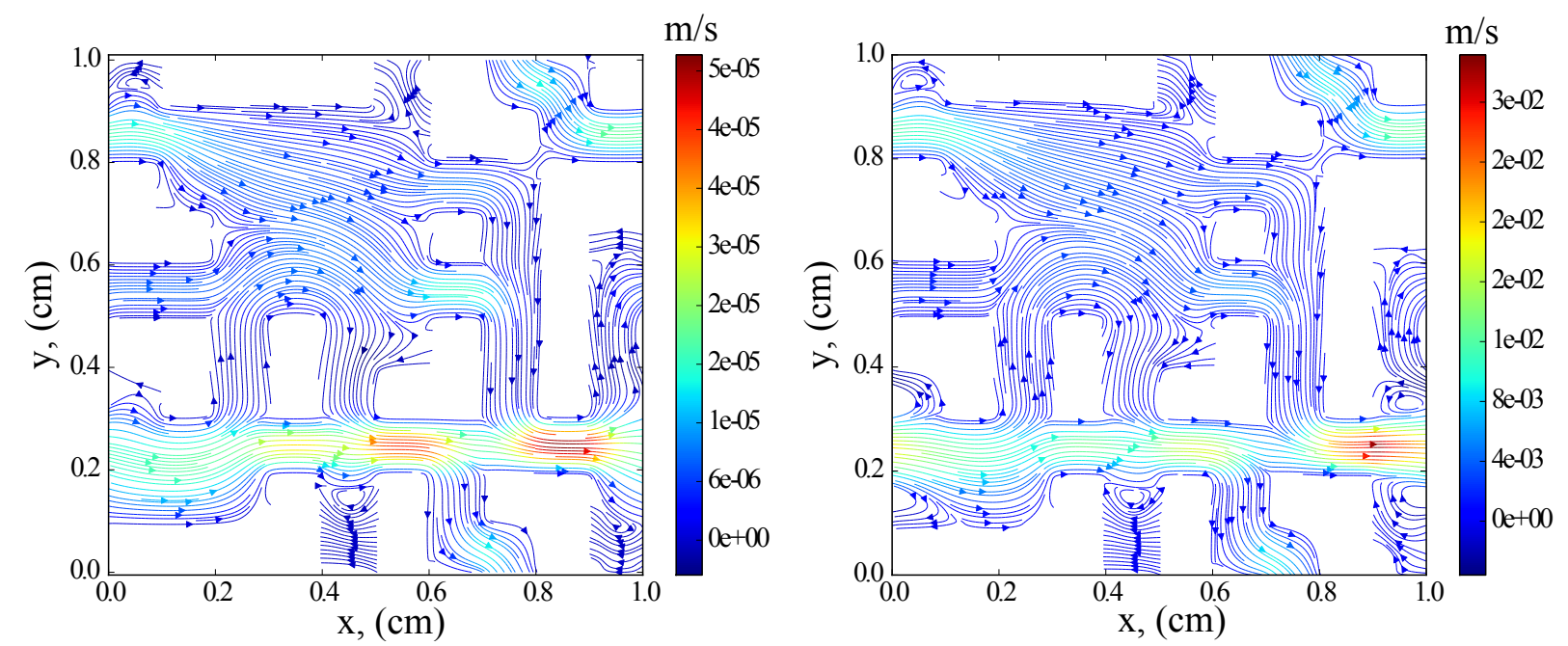

(a) Velocity streamlines for porosity (0.64) random

(b) Velocity streamlines for porosity (0.64) random $2 \mathrm{D}$ porous media with $F_{o}=4.87 \times 10^{-3}$

$2 \mathrm{D}$ porous media with $F_{o}=1.96 \times 10^{-1}$

Figure 4.23: Velocity streamlines for porosity (0.64) random $2 \mathrm{D}$ porous media $F_{o}=4.87 \times$ $10^{-3}$ (left) and $F_{o}=1.96 \times 10^{-1}$ (right)

Figure 4.24.a shows the plot of the apparent permeability against the Forchheimer numbers. It shows that there is a reduction of apparent permeability from $3.82 \times 10^{-7} \mathrm{~m}^{2}$ at $F_{o}=1.42 \times 10^{-4}$ to $1.84 \times 10^{-7} \mathrm{~m}^{2}$ at $F_{o}=7.89 \times 10^{-1}$ with (51.81 percent reduction). Figure 4.24.b shows a linear relationship between the inverse of apparent permeability and velocity over kinematic viscosity, $\frac{u}{\nu}$ with $R^{2}=0.9993$ and a non-Darcy factor of $525.72 \mathrm{~m}^{-1}$.

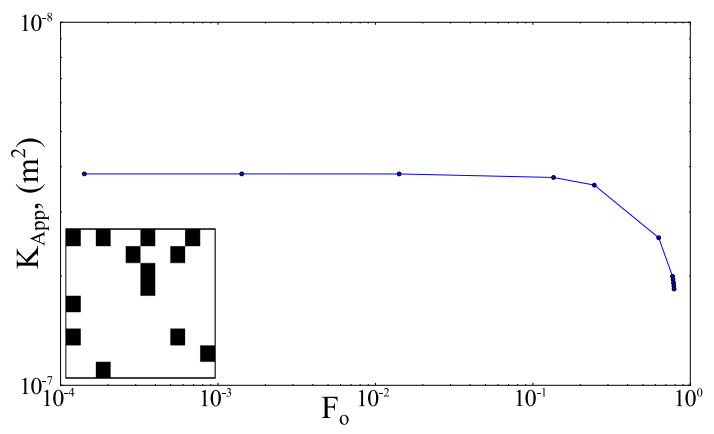

(a) Apparent permeability versus Forchheimer

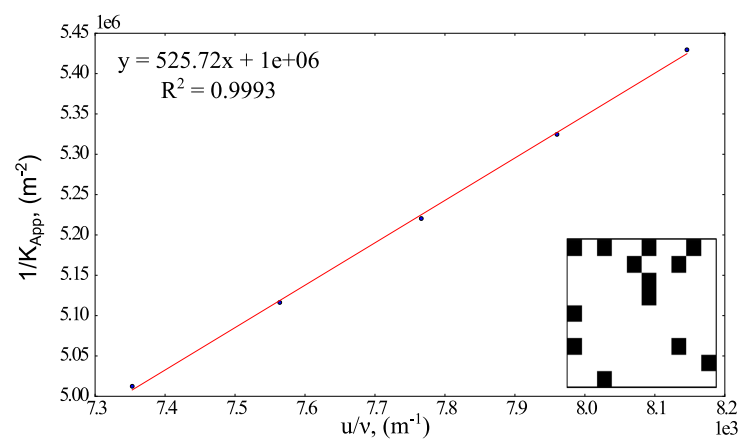

(b) Inverse apparent permeability versus $\mathrm{u} / \nu$ number for random porous media with 0.87 porosity for random porous media with 0.87 porosity

Figure 4.24: Simulations results of 2-dimensional random porous media for 0.87 porosity 
Figure 4.25.a shows that the velocity streamlines for porosity (0.87) random 2D porous media at $F_{o}=1.42 \times 10^{-4}$ are smooth and parallel. Figure 4.25.b shows some eddies in velocity streamline plot for $F_{o}=7.89 \times 10^{-1}$ due to the acceleration/deceleration of the fluid flow.
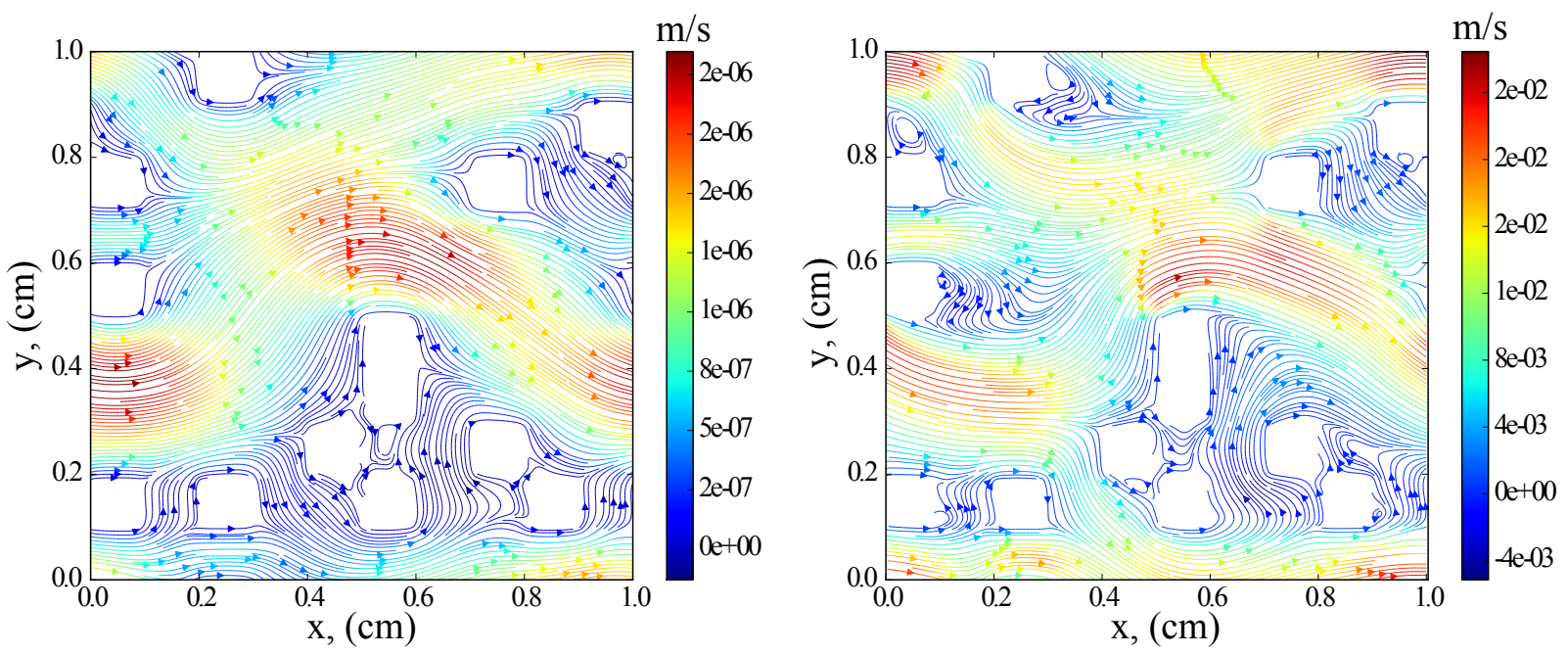

(a) Velocity streamlines for porosity (0.87) random

(b) Velocity streamlines for porosity (0.87) random $2 \mathrm{D}$ porous media with $F_{o}=1.42 \times 10^{-4}$

$2 \mathrm{D}$ porous media with $F_{o}=7.89 \times 10^{-1}$

Figure 4.25: Velocity streamlines for porosity (0.87) random $2 \mathrm{D}$ porous media $F_{o}=1.42 \times$ $10^{-4}$ (left) and $F_{o}=7.89 \times 10^{-1}$ (right)

Figure 4.26.a shows the plot of the apparent permeability against the Forchheimer numbers. It shows that there is a reduction of apparent permeability from $1.18 \times 10^{-8} \mathrm{~m}^{2}$ at $F_{o}=1.03 \times 10^{-4}$ to $1.06 \times 10^{-8} \mathrm{~m}^{2}$ at $F_{o}=2.01 \times 10^{-1}$ with $(9.70$ percent reduction $)$. Figure 4.26.b shows a linear relationship between the inverse of apparent permeability and velocity over kinematic viscosity, $\frac{u}{\nu}$ with $R^{2}=0.9995$ and a non-Darcy factor of $5012.2 \mathrm{~m}^{-1}$. 


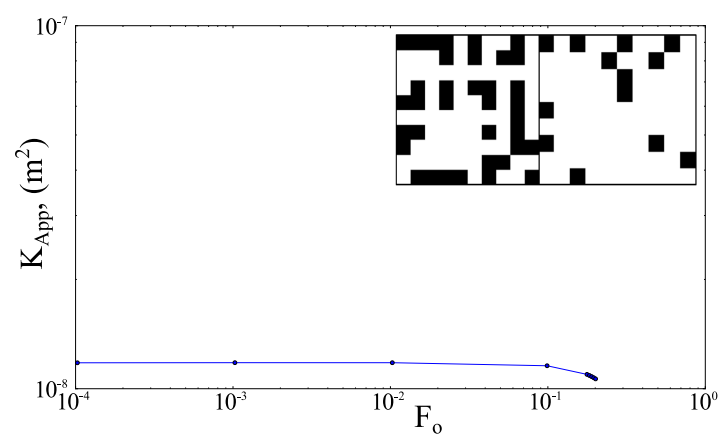

(a) Apparent permeability versus Forchheimer number for random porous media with low and high porosity

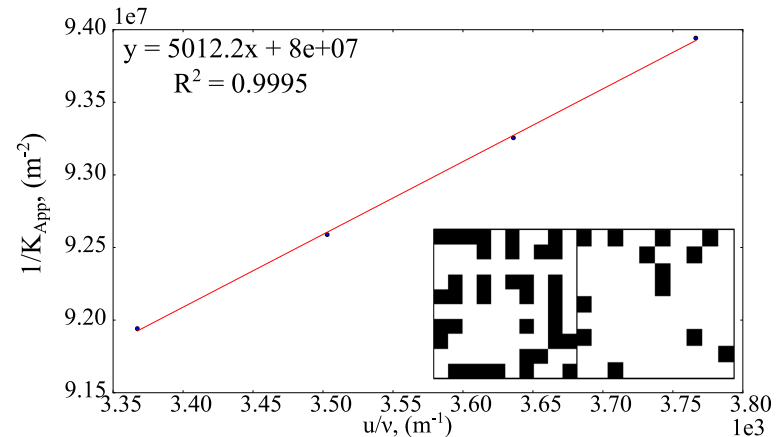

(b) Inverse apparent permeability versus $\mathrm{u} / \nu$ for random porous media with low and high porosity

Figure 4.26: Simulations results of 2-dimensional random porous media for low and high porosity

Figure 4.27.a shows that the velocity streamlines for low and high porosity 2D random porous media at $F_{o}=1.03 \times 10^{-4}$ are smooth and parallel. Figure 4.27.b shows some eddies in velocity streamline plot for $F_{o}=2.01 \times 10^{-1}$ due to the acceleration/deceleration of the fluid flow. Eddies are generated mostly in the interface between the randomly generated porous media of low and high porosity.

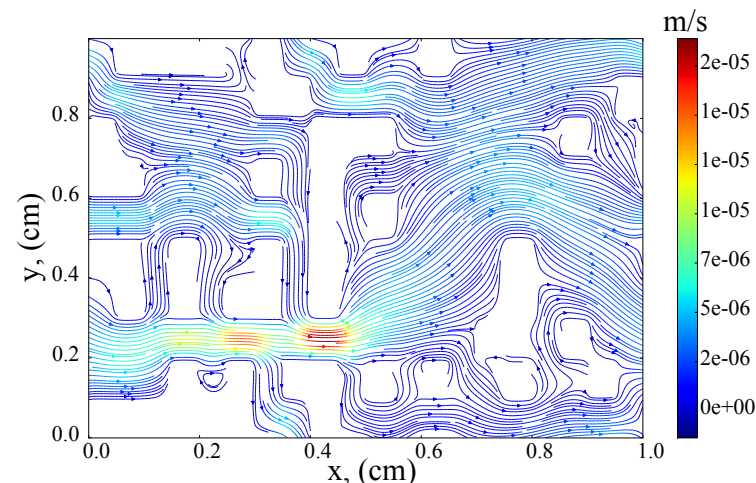

(a) Velocity streamlines for low and high porosity $2 \mathrm{D}$ random porous media with $F_{o}=1.03 \times 10^{-4}$

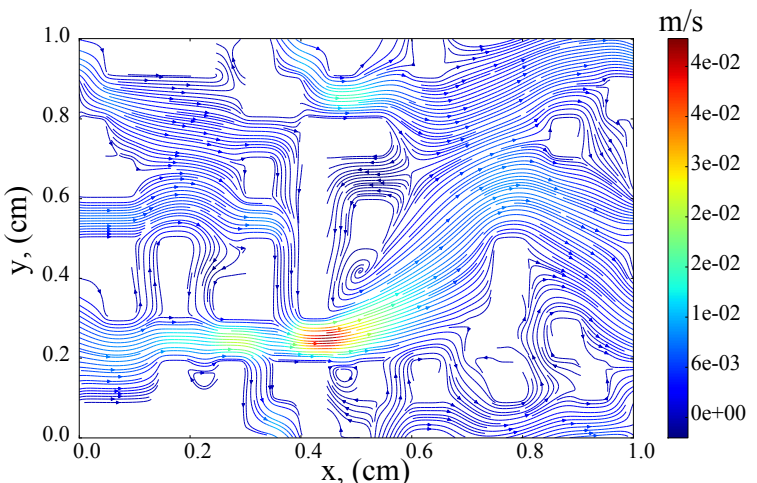

(b) Velocity streamlines for low and high porosity $2 \mathrm{D}$ random porous media with $F_{o}=2.01 \times 10^{-1}$

Figure 4.27: Velocity streamlines for low and high porosity $2 \mathrm{D}$ random porous media $F_{o}=$ $1.03 \times 10^{-4}$ (left) and $F_{o}=2.01 \times 10^{-1}($ right $)$ 


\subsection{Discussion}

Table 4.1 summarizes the results of the simulations for each geometry, as well as the calculated harmonic averages for the permeability using the following equation,

$$
\frac{\Sigma L_{i}}{K_{\text {total }}}=\Sigma \frac{L_{i}}{K_{i}}
$$

where $L_{i}$ is the length of the channel and $K_{i}$ is the permeability for the geometries, while $K_{\text {total }}$ is the permeability for the combined geometry.

\begin{tabular}{||cccc||}
\hline Geometry & $K$ & $K_{\text {total }}$ & $\beta$ \\
\hline \hline$(1 \times 1)$ & 123.2824 & - & 14.765 \\
$(2 \times 2)$ & 31.63985 & - & 69.427 \\
$(3 \times 3)$ & 14.32196 & - & 136.03 \\
$(4 \times 4)$ & 7.822869 & - & 302.73 \\
$(1 \times 1)+(2 \times 2)$ & 49.00067 & 50.3560 & 232.32 \\
$(1 \times 1)+(3 \times 3)$ & 25.08423 & 25.6626 & 345.75 \\
$(1 \times 1)+(4 \times 4)$ & 14.53737 & 14.7121 & 393.09 \\
$(2 \times 2)+(3 \times 3)$ & 19.27202 & 19.7183 & 317.9 \\
$(2 \times 2)+(4 \times 4)$ & 12.36359 & 12.5442 & 379.12 \\
$(3 \times 3)+(4 \times 4)$ & 9.981599 & 10.1187 & 440.44 \\
Low porosity & 1.647911 & - & 3981.0 \\
High porosity & 24.45982 & - & 525.72 \\
Low porosity + High porosity & 3.017687 & 3.0878 & 5012.2 \\
\hline
\end{tabular}

Table 4.1: Summary of the LBM simulation results

The permeability values for combined geometries are calculated by harmonic averaging of the permeability of two single geometries. This is an example to illustrate the value of $K_{\text {total }}$ based on the permeability values from the simulations of two single geometries.

$(1 \times 1)+(2 \times 2) \quad: \quad$ A combined geometry where $K($ simulation $)=49.00067$

$(1 \times 1) \quad:$ A single geometry where $K_{1}($ simulation $)=123.2824$

$(2 \times 2) \quad:$ A single geometry where $K_{2}($ simulation $)=31.63985$

$$
\frac{2}{K_{\text {total }}}=\frac{L}{123.2824}+\frac{L}{31.63985}=0.03972 \Rightarrow K_{\text {total }}=50.3560
$$

based on the final value of $K_{\text {total }}$, the value of the calculated permeability through series of constituting porous media follows the harmonic averaging theory. 
The non-Darcy factor for the geometry that is constructed by combining two single geometries are higher than their values for each of the single geometries. This confirms that the inertial effect has been influenced by of the acceleration/deceleration of the fluid at the interface of coarse-fine geometries. Furthermore, the obtained values of the non-Darcy factor does not follow any averaging technique (averaging values of two combined geometries are always between the value of the combined geometries). The non-Darcy factor is larger in a combined geometry than each of the two single geometries indirectly due to the fact that when the fluids in the porous media flow from tight to coarse pore space or vice verca, eddies could be generated due to the acceleration/deceleration of the fluid. The eddies occur when the fluid flows past an obstacle in high velocity which create swirling behind the two edges of the obstacle after passing it. Those swirling, which are created behind the obstacle, are flowing into the opposite direction of the general fluid flow direction such as figure 4.28.

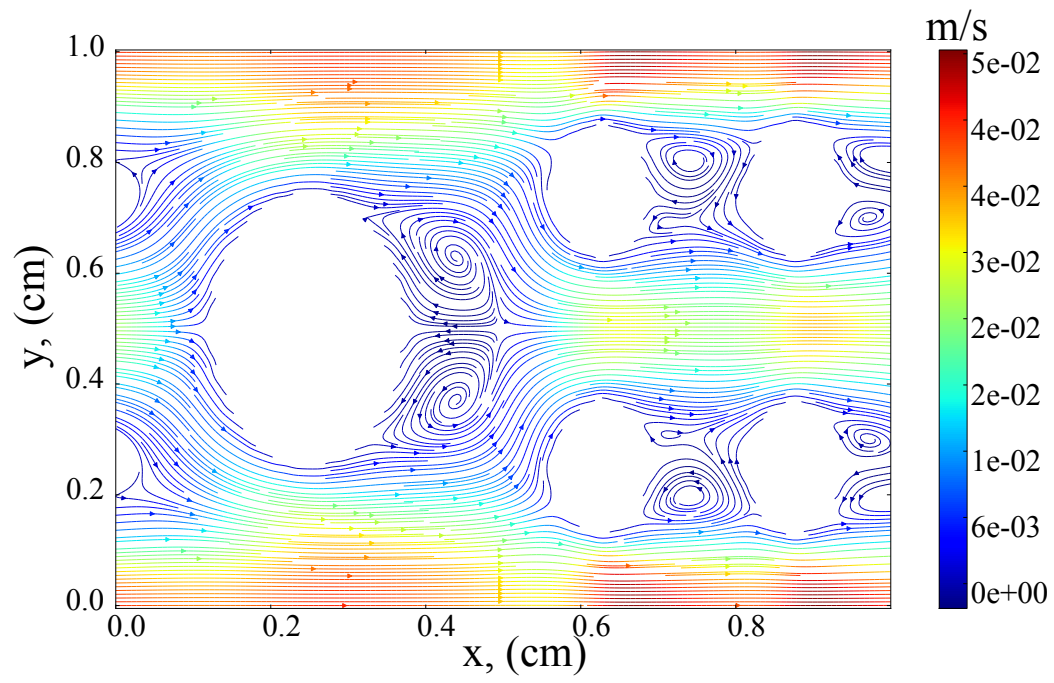

Figure 4.28: Eddies generated at the interface between fine and coarse geometries

The eddies in the porous media carry the most momentum and energy of the fluid flow [14] which causes the non-Darcy factor to be greater in the combined geometry than the single geometries which takes place mostly at the void spaces when the fluids flow though the interface of fine-coarse grids in the porous media. 


\section{Chapter 5}

\section{Conclusions and Recommendations}

\subsection{Conclusions and Recommendations}

In this work, LBM was used to obtain flow properties of 2 dimensional geometries at different Forchheimer numbers. The geometries were either simple (circle packs with single circle daimeter or random porous media with a certain porosity) or complex (created by combining the simple geometries)

Based on the simulation results of 2 dimensional circle packs, the higher circle diameter the lower non-Darcy factor. This is because fluids are not accelerating in bigger porous as much as they do in smaller porous. The non-Darcy factor for combined geometries resulted in higher values than simple geometries due to the acceleration/deceleration of the fluid flow from fine to coarse circle packs and vice versa which generates eddies at the interface.

The permeability obtained from the flow simulation for combined circle packs follows the theory of harmonic averaging. However, the values of non-Darcy factor obtained from flow simulations for combined circle packs are higher than those for the single circle packs; therefore, calculated non-Darcy factor do not follow any averaging theories which confirms that the non-Darcy factor cannot be upscalled. 


\subsection{Recommendations}

For future work, it is recommended to apply flow simulations to 3 dimensional geometries. The simulation results can also be inputted to a reservoir simulators to observe the impact of higher non-Darcy factor at the fracture-reservoir rock interface on the productivity index of the wells. Moreover, it is recommended to apply grid refinement technique because it reduces the time of the flow simulations by dividing the combined geometries into two different mesh sizes for proppant and for reservoir rock. 


\section{References}

[1] Zhengwen Zeng and Reid Grigg, "A Criterion for Non-Darcy Flow in Porous Media," Transport in Porous Media, vol. 63, no. 1, pp. 57-69, Apr. 2006.

[2] George V Chilingarian, Sal J Mazzullo, and Herman H Rieke, Carbonate reservoir characterization: a geologic-engineering analysis, Elsevier Science Ltd, 1996.

[3] SA Holditch and RA Morse, "The effects of non-darcy flow on the behavior of hydraulically fractured gas wells," Journal of Petroleum Technology, vol. 28, no. 10, pp. 1169-1179, 1976.

[4] Kern H Guppy, H Cinco-Ley, HJ Ramey Jr, et al., "Pressure buildup analysis of fractured wells producing at high flow rates," Journal of Petroleum Technology, vol. 34, no. 11, pp. 2-656, 1982.

[5] Michael C Sukop and Daniel T Thorne, Lattice Boltzmann modeling: an introduction for geoscientists and engineers, Springer, 2007.

[6] Johannes Habich, "Improving computational efficiency of lattice boltzmann methods on complex geometries," 2006.

[7] Jonas Latt, "Choice of units in lattice boltzmann simulations," Freely available online at http://lbmethod. org/_media/howtos: lbunits. pdf, 2008.

[8] Glenn O Brown, Jürgen D Garbrecht, and Willi H Hager, Henry PG Darcy and Other Pioneers in Hydraulics, ASCE Publications, 2003.

[9] J Geertsma et al., "Estimating the coefficient of inertial resistance in fluid flow through porous media," Society of Petroleum Engineers Journal, vol. 14, no. 05, pp. 445-450, 1974.

[10] Zhengwen Zeng and Reid Grigg, "A criterion for non-darcy flow in porous media," Transport in Porous Media, vol. 63, no. 1, pp. 57-69, 2006.

[11] Shiyi Chen and Gary D Doolen, "Lattice boltzmann method for fluid flows," Annual review of fluid mechanics, vol. 30, no. 1, pp. 329-364, 1998. 
[12] JA Rushing, KE Newsham, PM Lasswell, JC Cox, TA Blasingame, et al., "Klinkenergcorrected permeability measurements in tight gas sands: Steady-state versus unsteadystate techniques," in SPE Annual Technical Conference and Exhibition. Society of Petroleum Engineers, 2004.

[13] CC Mei and J-L Auriault, "The effect of weak inertia on flow through a porous medium," Journal of Fluid Mechanics, vol. 222, pp. 647-663, 1991.

[14] Marcelo JS De Lemos, Turbulence in porous media: modeling and applications, Elsevier, 2012. 